See discussions, stats, and author profiles for this publication at: https://www.researchgate.net/publication/333830619

\title{
Bounded and Compact Weighted Essentially Nonoscillatory Limiters for Discontinuous Galerkin Schemes: Triangular Elements
}

Article in Journal of Computational Physics · June 2019

DOI: 10.1016/.j.jp. 2019.06.023

\section{CITATIONS}

3 authors, including:

Alireza Mazaheri

NASA

47 PUBLICATIONS 346 CITATIONS

SEE PROFILE

Some of the authors of this publication are also working on these related projects:

Multiphase compressible flows View project

Project

Extending the proposed DG-H schemes to Navier-Stokes View project
READS

69

Vincent Perrier

National Institute for Research in Computer Science and Control 39 PUBLICATIONS 166 CITATIONS

SEE PROFILE 


\title{
Bounded and compact weighted essentially nonoscillatory limiters for discontinuous Galerkin schemes: Triangular elements
}

\author{
Alireza Mazaheri ${ }^{\mathrm{a}, *, 1}$, Chi-Wang Shu ${ }^{\mathrm{b}, 2}$, Vincent Perrier ${ }^{\mathrm{c}, 3}$ \\ a Aerothermodynamics Branch, Research Directorate, NASA Langley Research Center, Hampton, VA 23681, USA \\ b Division of Applied Mathematics, Brown University, Providence, RI 02912, USA \\ ' INRIA Bordeaux Sud-Ouest, Laboratoire de Mathématiques et de leurs applications Bâtiment IPRA, Universite de Pau et des Pays de l'Adour, \\ 64013 Pau Cedex, France
}

\section{A R T I C L E I N F O}

\section{Article history:}

Received 28 May 2018

Received in revised form 28 March 2019

Accepted 8 June 2019

Available online $\mathrm{xxxx}$

\section{Keywords:}

CWENO

Unstructured meshes

High-order DG

Positivity-preserving

Riemann problem

Shu-Osher

\begin{abstract}
A B S T R A C T
Two new classes of compact weighted essentially nonoscillatory (WENO) polynomial limiters are presented for second-, third-, fourth-, and fifth-order discontinuous Galerkin (DG) schemes on irregular simplex elements. The presented WENO-DG procedures are extensions of the high-order WENO finite-volume and finite-difference schemes of Zhu and Shu (2017) [25], (2019) [26] to high-order unstructured DG schemes. A compact positivity preserving limiter is applied to the solutions to ensure pressure and density remain within physical ranges at all time. It is then verified that the bounded WENODG maintains the formal order of accuracy of the underlying DG schemes in the smooth regions. The performance of the proposed WENO-DG is also demonstrated with inviscid test cases including the classical Riemann problems, shock-turbulence interaction, scramjet, blunt body flows, and the double Mach Reflection problems.
\end{abstract}

Published by Elsevier Inc

\section{Introduction}

Consider the unsteady compressible Euler equations for a perfect gas flow

$$
\mathbf{w}_{t}+\nabla \cdot \mathbf{f}(\mathbf{w})=0, \quad \mathbf{w}(\mathbf{x}, t=0)=\mathbf{w}_{0}(\mathbf{x}),
$$

where

$$
\mathbf{w}=\left(\begin{array}{c}
\rho \\
\rho \mathbf{u} \\
\rho E
\end{array}\right), \quad \mathbf{f}(\mathbf{w})=\left(\begin{array}{c}
\rho \mathbf{u} \\
\rho \mathbf{u} \mathbf{u}^{T}+p \mathbf{I} \\
\rho \mathbf{u} H
\end{array}\right), \quad H=E+\frac{p}{\rho},
$$

\footnotetext{
* Corresponding author.

E-mail addresses: alireza.mazaheri@nasa.gov (A. Mazaheri), shu@dam.brown.edu (C.-W. Shu), vincent.perrier@inria.fr (V. Perrier).

1 Research supported by the NASA Langley CIF/IRAD project, and the NASA STMD ESM/GCD program.

2 Research supported by ARO grant W911NF-15-1-0226 and NSF grant DMS-1719410.

3 Research supported by NASA Langley CIF/IRAD project while visiting NASA Langley from January - March 2017.

https://doi.org/10.1016/j.jcp.2019.06.023

0021-9991/Published by Elsevier Inc.
} 
$\rho$ is density, $\mathbf{u}$ is the velocity vector in $\mathbf{x}$ directions, $\mathbf{I}$ is an identity matrix, and the total energy, $\rho E$, is defined as

$$
\rho E=\frac{p}{\gamma-1}+\frac{1}{2} \rho\|\mathbf{u}\|^{2}
$$

where $p$ is the pressure and $\gamma$ is the specific heat ratio.

In the present work, Eq. (1) may be spatially discretized with high-order discontinuous Galerkin (DG) schemes on triangular elements, and applied to continuous and discontinuous flows. The temporal term may be discretized with either the three stage, third-order strong stability preserving $\operatorname{SSP}(3,3)$ Runge-Kutta (RK) $[1,2]$, or the backward Euler time discretization method.

As with many nonlinear partial differential equations, the solution of Eq. (1) may contain a strong discontinuity with abrupt changes in physical quantities, such as density, pressure, and temperature. The DG method produces accurate results if the solution is smooth and/or contains (relatively) weak discontinuities, otherwise significant oscillations and/or nonlinear instabilities may occur. To avoid such difficulties with numerical oscillations, the DG method needs to be accompanied by, for example, a limiter strategy such as minmod [3-6], artificial viscosity [7-10], total variation diminishing [11-13], or weighted essentially nonoscillatory (WENO) [14-19] techniques. The advantage of WENO type limiters is that they can be compact, of high order, and more importantly do not affect the expected order of accuracy of the underlying DG scheme if applied to smooth regions. Available WENO schemes are either non-compact $[20,21,14,22,16,15,23,24]$, which is undesirable for DG methods, or have difficulties in reducing the residuals $[20,21,24]$. The proposed WENO-FV and WENO-FD schemes of Zhu and Shu $[25,26]$ address the convergence difficulties that are observed in the earlier WENO procedures [27,28].

Advancement in limiting procedures have also been reported for unstructured simplex elements. For instance, Dumbser and Loubère [29] proposed a posteriori subcell finite volume limiter for DG schemes on simplex elements, while a compact WENO limiter for ADER-FV schemes on fixed and moving simplex elements is proposed by Dumbser et al. [30].

Here, two classes of compact WENO polynomial limiters are presented for DG methods on simplex elements that may be considered extensions of the WENO-FV [25] and WENO-FD [31] schemes. Although the original compact WENO limiters for finite volume and finite difference schemes rely on an extended stencil for polynomial reconstruction, the main idea behind the compact WENO for DG schemes is to construct a limited polynomial directly from the underlying DG scheme. The compact WENO-DG limiters presented here, therefore, require a stencil only as large as (nfaces) ${ }^{(0)}+1$ number of elements, where (nfaces) ${ }^{(0)}$ corresponds to the number of faces of the elements in which the limiter is being applied. For example, for triangles, the compact WENO stencil has only up to 4 elements (see also Fig. 1). In the first proposed approach, the compact stencil is considered, and a series of linear polynomials is constructed by solving a (dof $\times$ dof) linear system, where dof denotes degrees of freedom. In the second approach, a linear polynomial is constructed by solving a series of constrained least-squares (LSQ) minimization problems within the compact stencil. In both approaches, a WENO polynomial limiter is obtained with a convex combination of the original polynomial and the constructed linear polynomials that are added using nonlinear weights. The numerical fluxes are evaluated with the Local Lax-Friedrichs (LLxF) flux, and a compact positivity preserving limiter [32] is also applied to the solutions to ensure pressure and density remain bounded and physical at all time.

The paper is organized as follows. Section 2 describes in detail the proposed compact WENO polynomial limiters for unstructured DG schemes with simplex elements. The positivity preserving limiter for the Euler equations is briefly discussed in Section 3. A few sample problems, ranging from the classical Sod and Lax problems to blunt body flows and double Mach reflection test case are presented in Section 4. The concluding remarks are given in Section 5.

\section{Proposed WENO-DG polynomial limiters}

Two new classes of compact WENO polynomial limiters are proposed here for unstructured DG schemes. These schemes are in part inspired by the recent successful WENO finite difference and finite volume procedures of Zhu and Shu [25,26], which are extensions and steady state computations of the earlier works reported by Zhu and Qiu [28,33,34,25] and Levy et al. [27]. The proposed WENO procedures are designed for simplex elements, and are compact, requiring information only from the immediate neighbors. For each presented approach, a detailed step-by-step procedure is outlined that may be used for implementation of the WENO as a post-processing step to the DG solution after each time iteration.

Consider a compact triangular stencil $\{0,1,2,3\}$ as shown in Fig. 1, where the element 0 is the target element for which the limiter is applied. The goal is to reconstruct a new candidate polynomial for the target element, $p^{(0) n e w}$, that is nonoscillatory in the presence of discontinuities, and dependent only on the available information within the compact stencil. We remark that the polynomials constructed throughout this document are defined with a set of basis functions expressed in a reference element.

The original polynomial on the target element, $p^{(0)}$, may be expressed in the following identity relation as

$$
\tilde{p}^{(0)}:=p^{(0)}=\gamma_{0}\left(\frac{1}{\gamma_{0}} p^{(0)}-\sum_{i} \frac{\gamma_{i}}{\gamma_{0}} \tilde{p}^{(i)}\right)+\sum_{i} \gamma_{i} \tilde{p}^{(i)}
$$

which holds for arbitrary positive coefficients $\gamma_{j}$ satisfying $\sum_{j=0}^{3} \gamma_{j}=1$, where, $\gamma_{j}$, are the linear weights defined as 


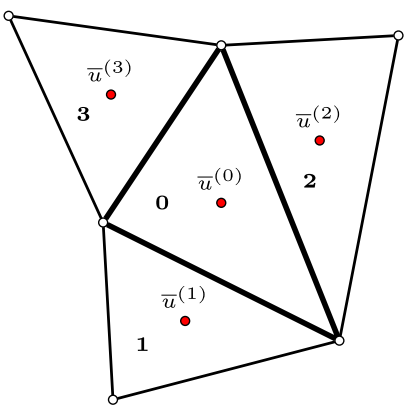

(a) interior

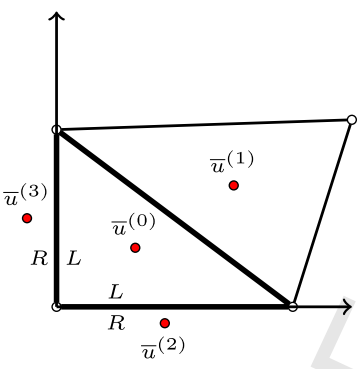

(b) boundary

Fig. 1. The compact WENO-DG stencil for triangular elements.

$$
\gamma_{0}=0.997, \quad \gamma_{j}=0.001(j \neq 0),
$$

and the $\tilde{p}^{(i)}$ are the to-be-reconstructed polynomials for the elements $i=1,2,3$ (i.e., the immediate neighbors of the target element). The $\tilde{p}^{(i)}$ reconstruction procedures are discussed in detail later. The linear weights are then replaced with the nonlinear weights, $\omega$, and the following reconstructed candidate polynomial is sought for the target element ${ }^{4}$

$$
p^{(0) \text { new }}=\omega_{0}\left(\frac{1}{\gamma_{0}} p^{(0)}-\sum_{i} \frac{\gamma_{i}}{\gamma_{0}} \tilde{p}^{(i)}\right)+\sum_{i} \omega_{i} \tilde{p}^{(i)}
$$

where the nonlinear weights are computed by adopting the high-order WENO-FD [25] and WENO-FV [26] techniques of Zhu and Shu for the present WENO-DG schemes. These techniques are based on the WENO-Z strategy given in Refs. [35-37]. The nonlinear weights are then defined as

$$
\omega_{i}=\frac{\bar{\omega}_{i}}{\sum_{j} \bar{\omega}_{j}}, \quad \bar{\omega}_{j}=\gamma_{j}\left(1+\frac{\tau}{\left(\epsilon+\beta_{j}\right)^{2}}\right), \quad \tau=\left(\frac{\sum_{i}\left|\beta_{0}-\beta_{i}\right|}{n}\right)^{2},
$$

where $n$ is the total number of reconstructed polynomials used in constructing the WENO polynomial $p^{(0) \text { new }}$ as given in Eq. (5) (i.e., maximum integer value of $i$ ) plus the polynomial of the target element $\Delta_{0}$. Here $\epsilon=10^{-12}$ is a small number to avoid zero denominator, and $\beta$ is the classical smoothness indicator defined as (see e.g., Jiang and Shu [20] and Kolb [38])

$$
\beta_{i}=\sum_{|l|=1}^{k}\left|\Delta_{0}\right|^{(|l|-1)} \int_{\Delta_{0}}\left(\frac{\partial^{|l|}}{\partial x^{l_{1}} \partial y^{l_{2}}} \tilde{p}^{(i)}\right)^{2}, \quad l=\left(l_{1}, l_{2}\right),
$$

where $k$ denotes the polynomial order, and $\left|\Delta_{0}\right|$ is the volume of the target element.

The above procedure is complete with reconstruction of the polynomials $\tilde{p}^{(i)}$. This is discussed next where two compact reconstruction approaches are presented in detail.

\subsection{Polynomial reconstruction approach 1}

In this section, a procedure to reconstruct a set of polynomials, $\tilde{p}^{(i)}$, is described in detail. The reconstructed polynomials are used for the WENO polynomial limiter given by Eq. (5). First, a procedure for a scalar case is given followed by a system case.

\subsubsection{Scalar case}

1. Compute the means of the polynomials on the compact WENO stencil $\{0,1,2,3\}$ :

$$
\bar{u}^{(i)}=\frac{1}{\left|\Delta_{i}\right|} \int_{\Delta_{i}} p^{(i)}, \quad i=0,1,2,3,
$$

where $\Delta_{i}$ denotes neighboring elements with corresponding volume of $\left|\Delta_{i}\right|$. For boundary elements, first obtain the right values $u^{R}$ by applying the boundary condition along the boundary face, and then integrate $u^{R}$ to get the mean values. If

\footnotetext{
${ }^{4}$ Classical trouble cell indicators are not employed in the presented examples and therefore, the proposed WENO is applied to all the computational elements. Hence, all the elements in the computational domain are considered as target elements. This approach is beneficial in ensuring that the proposed WENO does not affect the desired order of accuracy of the DG scheme even if it is artificially applied to smooth region.
} 
the boundary is an outflow, for example a supersonic outflow, on which we do nothing, integrate the left values $u^{L}$ on the boundary face to get the corresponding mean values.

2. Construct a set of linear polynomials, $\tilde{p}^{(j)}$, such that

$$
\frac{1}{\left|\Delta_{i}\right|} \int_{\Delta_{i}} \tilde{p}^{(j)}=\bar{u}^{(i)},
$$

where $i$ corresponds to the element number in the compact WENO stencil (see Fig. 1), and $j$ refers to the candidate linear polynomial number. For a triangle, we have three such candidates, and for each candidate, we use the elements in the $i$ th set to construct a linear polynomial $\tilde{p}^{(j)}$ :

$$
(j, i):=(1,\{0,1,2\}) ;(2,\{0,1,3\}) ;(3,\{0,2,3\}) .
$$

Remark. All the reconstructed linear polynomials are defined within the target element, with the basis functions, $\tilde{\varphi}$, also defined on the target element. Such basis functions are denoted as $\tilde{\varphi}_{i}^{(0)}$. For example, consider the $j=1$ polynomial, $\tilde{p}^{(1)}$. This is defined as

$$
\tilde{p}^{(1)}=\sum_{i=0}^{2} \tilde{u}_{i} \tilde{\varphi}_{i}^{(0)} .
$$

This polynomial is then used for evaluation of the integral given in Eq. (9) with appropriate quadrature rules. The elements in the $i$ th set $\{0,1,2\}$ are needed in the construction of the polynomial $\tilde{p}^{(1)}$. Therefore, a $3 \times 3$ linear system is solved to obtain the three coefficients (degrees of freedom) of the reconstructed linear polynomial. Thus, for the $j$ and $i$ th set used in this example, the following linear system is solved:

$$
\left(\begin{array}{ccc}
\left.\frac{1}{\left|\Delta_{0}\right|} \sum_{q} w_{q} \varphi_{0}^{(0)}\right|_{q} & \left.\frac{1}{\left|\Delta_{0}\right|} \sum_{q} w_{q} \varphi_{1}^{(0)}\right|_{q} & \left.\frac{1}{\left|\Delta_{0}\right|} \sum_{q} w_{q} \varphi_{2}^{(0)}\right|_{q} \\
\left.\frac{1}{\left|\Delta_{1}\right|} \sum_{q} w_{q} \varphi_{0}^{(0 \rightarrow 1)}\right|_{q} & \left.\frac{1}{\left|\Delta_{1}\right|} \sum_{q} w_{q} \varphi_{1}^{(0 \rightarrow 1)}\right|_{q} & \left.\frac{1}{\left|\Delta_{1}\right|} \sum_{q} w_{q} \varphi_{2}^{(0 \rightarrow 1)}\right|_{q} \\
\left.\frac{1}{\left|\Delta_{2}\right|} \sum_{q} w_{q} \varphi_{0}^{(0 \rightarrow 2)}\right|_{q} & \left.\frac{1}{\left|\Delta_{2}\right|} \sum_{q} w_{q} \varphi_{1}^{(0 \rightarrow 2)}\right|_{q} & \left.\frac{1}{\left|\Delta_{2}\right|} \sum_{q} w_{q} \varphi_{2}^{(0 \rightarrow 2)}\right|_{q}
\end{array}\right)\left(\begin{array}{c}
\tilde{u}_{0} \\
\tilde{u}_{1} \\
\tilde{u}_{2}
\end{array}\right)=\left(\begin{array}{c}
\bar{u}^{(0)} \\
\bar{u}^{(1)} \\
\bar{u}^{(2)}
\end{array}\right),
$$

where subscript $q$ denotes summation over the element quadrature points, $w_{q}$ corresponds to the quadrature weights, and the arrow indicates the extrapolation of the basis onto the neighbor elements 1 and 2 . The above system is valid for interior elements as shown in Fig. 1a. For elements that have faces on physical boundaries, the above system (12) takes a different form. For instance, consider $i$ th set $\left\{1, \partial \Omega_{1}, \partial \Omega_{2}\right\}$ where $\partial \Omega$ denotes the corresponding boundary face of the target element that is on a boundary. In this case, the system (12) reduces to

$$
\left(\begin{array}{ccc}
\left.\frac{1}{\left|\Delta_{0}\right|} \sum_{q} w_{q} \varphi_{0}^{(0)}\right|_{q} & \left.\frac{1}{\left|\Delta_{0}\right|} \sum_{q} w_{q} \varphi_{1}^{(0)}\right|_{q} & \left.\frac{1}{\left|\Delta_{0}\right|} \sum_{q} w_{q} \varphi_{2}^{(0)}\right|_{q} \\
\left.\frac{1}{\left|\partial \Omega_{1}\right|} \sum_{\partial q} w_{\partial q} \varphi_{0}^{(0)}\right|_{\partial q} & \left.\frac{1}{\left|\partial \Omega_{1}\right|} \sum_{\partial q} w_{\partial q} \varphi_{1}^{(0)}\right|_{\partial q} & \left.\frac{1}{\left|\partial \Omega_{1}\right|} \sum_{\partial q} w_{\partial q} \varphi_{2}^{(0)}\right|_{\partial q} \\
\left.\frac{1}{\left|\partial \Omega_{2}\right|} \sum_{\partial q} w_{\partial q} \varphi_{0}^{(0)}\right|_{\partial q} & \left.\frac{1}{\left|\partial \Omega_{2}\right|} \sum_{\partial q} w_{\partial q} \varphi_{1}^{(0)}\right|_{\partial q} & \left.\frac{1}{\left|\partial \Omega_{2}\right|} \sum_{\partial q} w_{\partial q} \varphi_{2}^{(0)}\right|_{\partial q}
\end{array}\right)\left(\begin{array}{c}
\tilde{u}_{0} \\
\tilde{u}_{1} \\
\tilde{u}_{2}
\end{array}\right)=\left(\begin{array}{c}
\bar{u}^{(0)} \\
\bar{u}_{\partial \Omega_{1}} \\
\bar{u}_{\partial \Omega_{2}}
\end{array}\right),
$$

where $\partial \Omega$ denotes the length of the corresponding boundary face, $\partial q$ indicates summation over the corresponding boundary face quadratures, and $\bar{u}_{\partial \Omega_{l}}$ is the mean value evaluated on the corresponding boundary face of the target element after the boundary condition is applied.

The same process is repeated to construct the other two linear polynomials $\tilde{p}^{(2)}$ and $\tilde{p}^{(3)}$.

3. Project the three basis functions of the constructed linear polynomials onto the basis space of the original polynomial of the target element, $p^{(0)}$; i.e., the $(k+d) ! /(k d !)$ basis functions, where $k$ is the polynomial order, and $d$ is the element dimension.

4. Reconstruct the new WENO limiter as given in Eq. (5) with $i=1,2,3$.

\subsubsection{System case}

1. Follow steps 1 and 2 given for the scalar case.

2. Project $\tilde{p}^{(j)}, j=0,1,2,3$ onto the characteristic fields

$$
\tilde{\tilde{p}}_{i}^{(j)}=L_{i} \cdot \tilde{p}^{(j)},
$$

where $L_{i}$ are the left eigenvectors based on the mean values of the target cell and the normal direction $i$. For a triangle, there are three normal directions corresponding to each face of the element. Note: $\tilde{p}^{(0)}:=p^{(0)}$. 


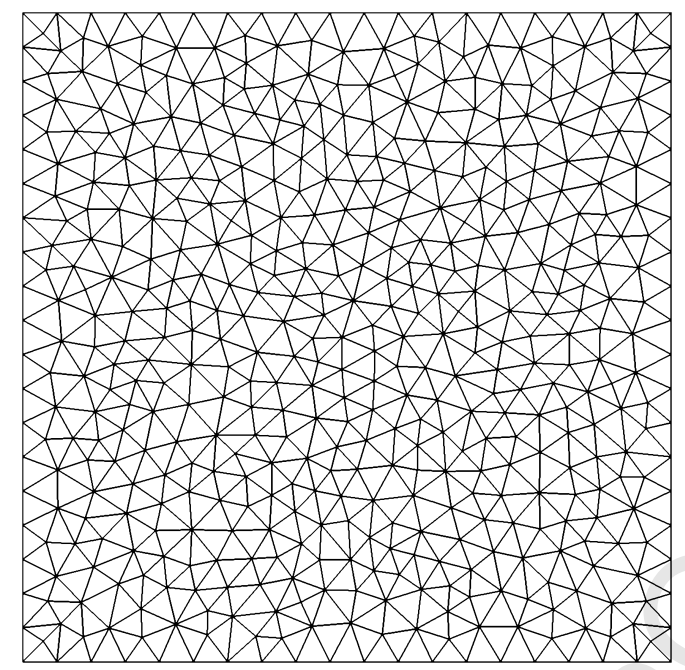

Fig. 2. A sample of randomly generated irregular triangular elements.

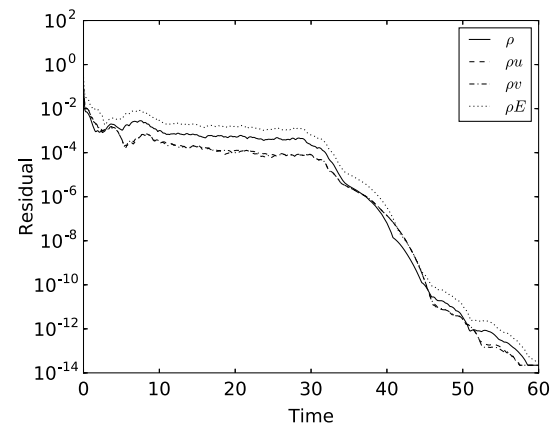

(a) $\mathrm{DG}(P 2)$

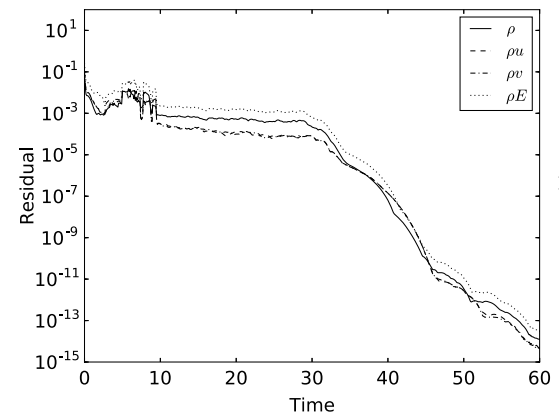

(d) WENO-DG(P2) (approach 1)

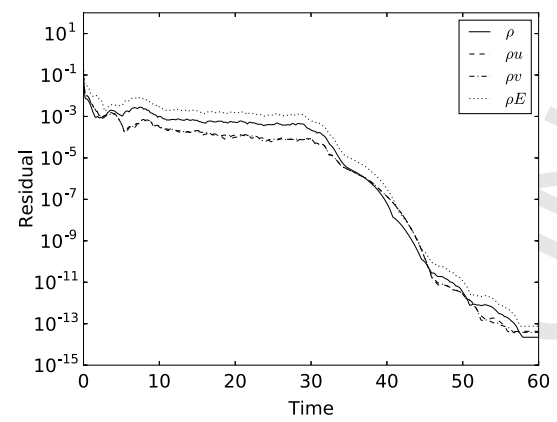

(g) WENO-DG $(P 2)$ (approach 2)

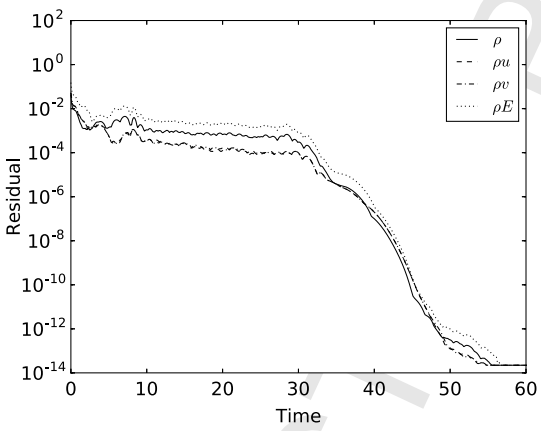

(b) $\mathrm{DG}(P 3)$

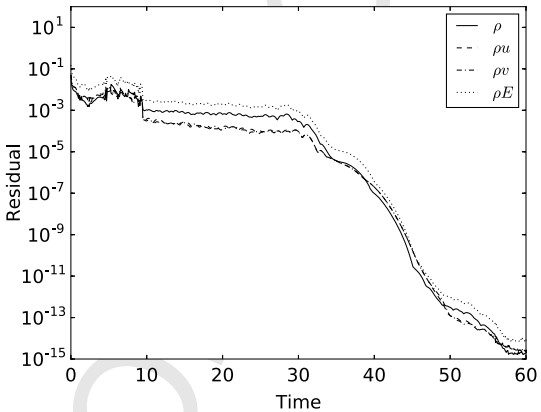

(e) WENO-DG(P3) (approach 1)

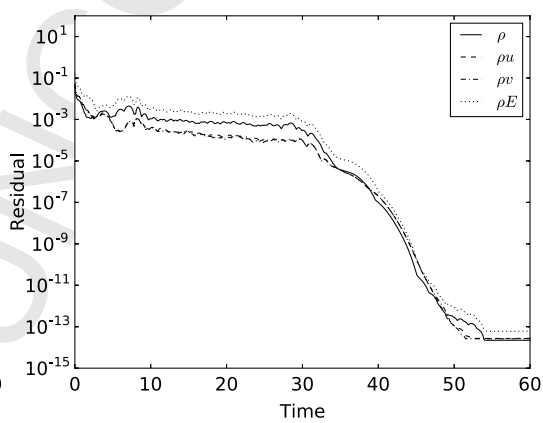

(h) WENO-DG(P3) (approach 2)

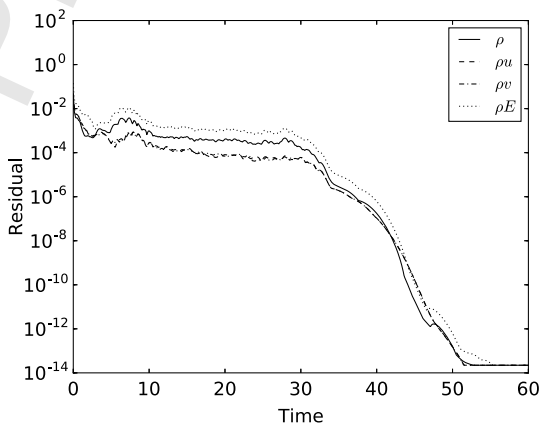

(c) $\mathrm{DG}(P 4)$

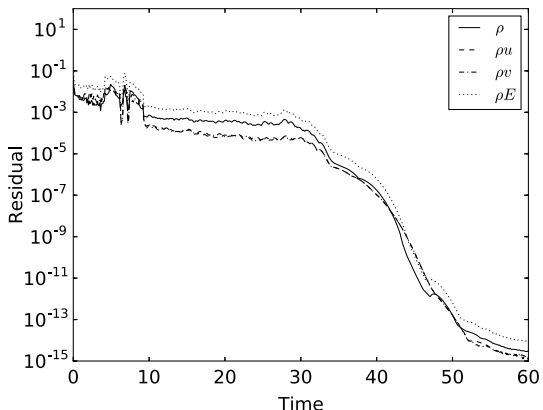

(f) WENO-DG(P4) (approach 1)

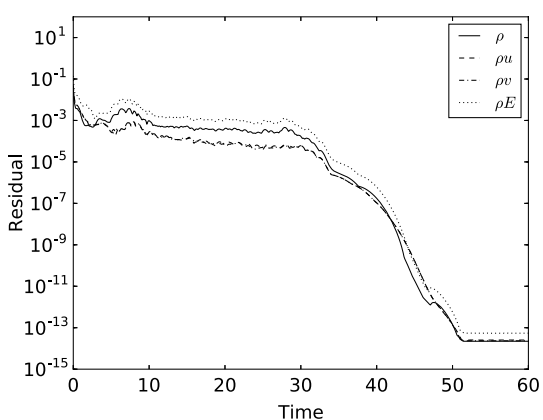

(i) WENO-DG $(P 4)($ approach 2)

Fig. 3. Verification - Residual history for the coarsest mesh. 


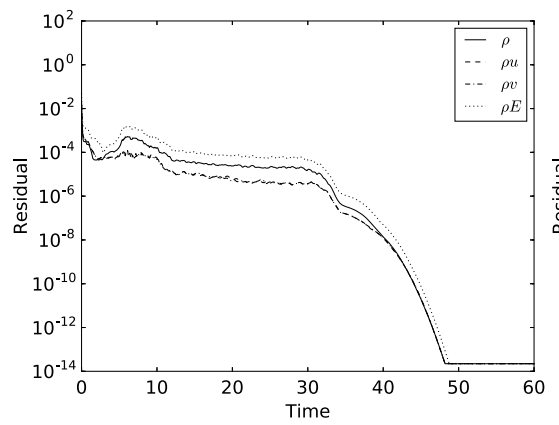

(a) $\mathrm{DG}(P 2)$

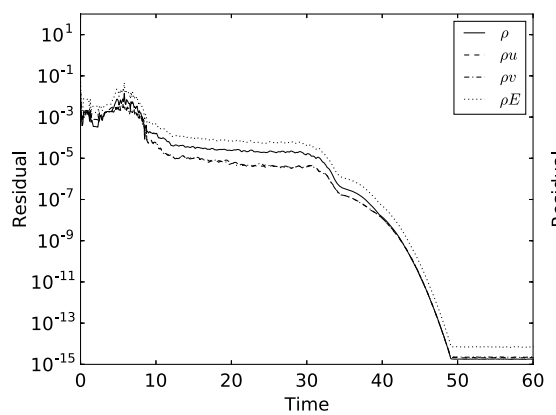

(d) WENO-DG(P2) (approach 1)

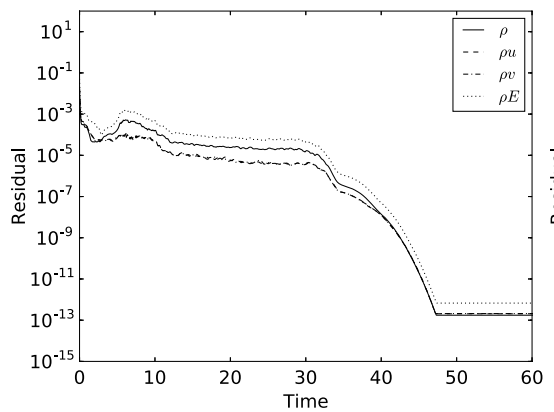

(g) WENO-DG(P2) (approach 2)

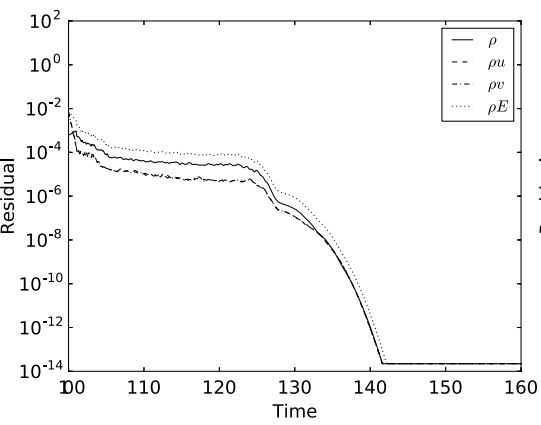

(b) $\mathrm{DG}(P 3)$

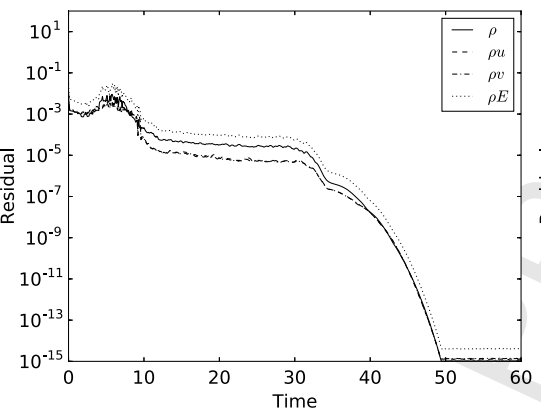

(e) WENO-DG(P3) (approach 1)

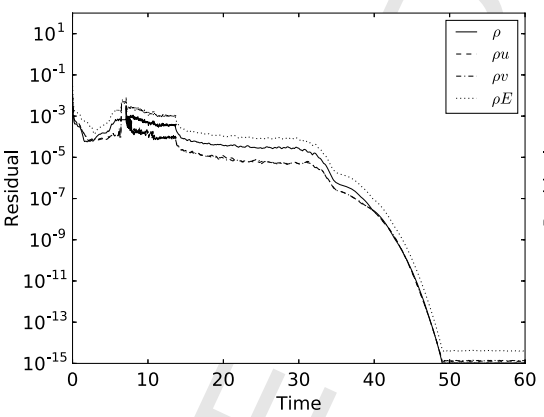

(h) WENO-DG(P3) (approach 2)

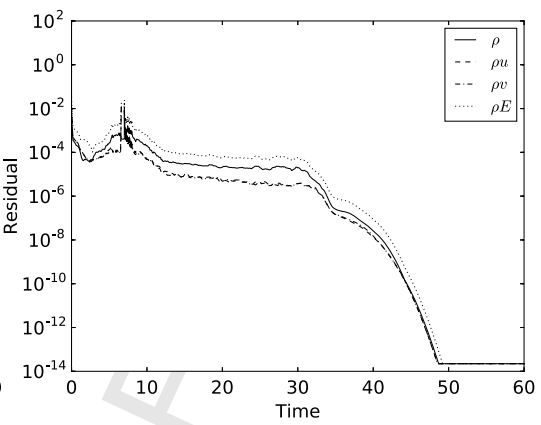

(c) $\mathrm{DG}(P 4)$

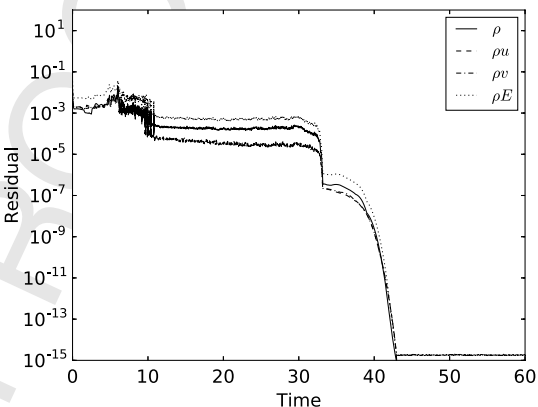

(f) WENO-DG(P4) (approach 1)

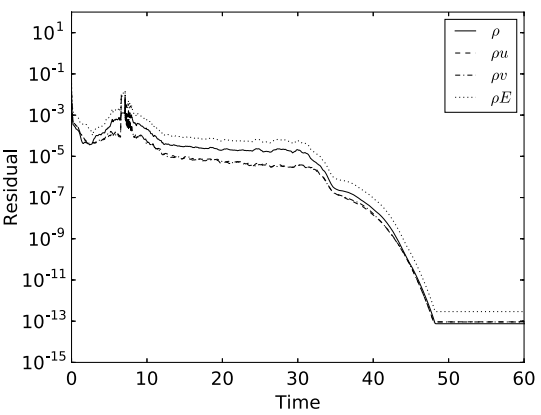

(i) WENO-DG(P4) (approach 2)

Fig. 4. Verification - Residual history for the finest mesh.

3. Use $\tilde{\tilde{p}}_{i}^{j}$, and follow steps 3 and 4 given for the scalar case, and evaluate $\tilde{p}_{i}^{(0)^{\text {new }}}$ for each direction $i$; i.e.,

$$
\tilde{p}_{i}^{(0)^{\text {new }}}=\frac{\omega_{0}}{\gamma_{0}} \tilde{\tilde{p}}_{i}^{(0)}+\sum_{j}\left(\omega_{j}-\omega_{0} \frac{\gamma_{j}}{\gamma_{0}}\right) \tilde{\tilde{p}}_{i}^{(j)}, \quad j=1,2,3, \quad i=1,2,3 .
$$

4. Project $\tilde{p}_{i}^{(0)^{\text {new }}}$ back into the physical space

$$
p_{i}^{(0)^{\text {new }}}=R_{i} \cdot \tilde{p}_{i}^{(0)^{\text {new }}},
$$

where $R_{i}$ are the right eigenvectors based on the mean values of the target cell and the normal direction $i$.

5. Obtain the final reconstructed WENO polynomial for the target cell with weighted averaging:

$$
p^{(0)^{\text {new }}}=\frac{\sum_{i} p_{i}^{(0)^{\text {new }}}\left|\Delta_{i}\right|}{\sum_{i}\left|\Delta_{i}\right|}
$$

where $\left|\Delta_{i}\right|$ corresponds to the volume of the neighboring element for face $i$ of the target element. If the face is a boundary face, use the volume of the target element, $\left|\Delta_{0}\right|$. 


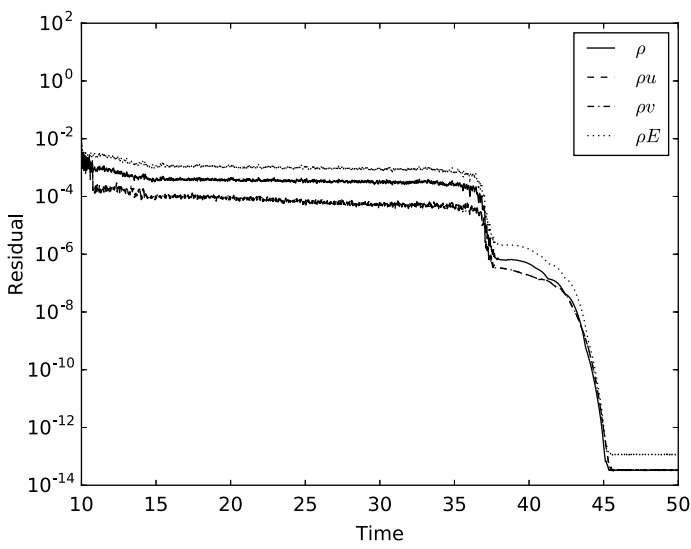

(a) Explicit; $\operatorname{SSP}(3,3) \mathrm{RK}$

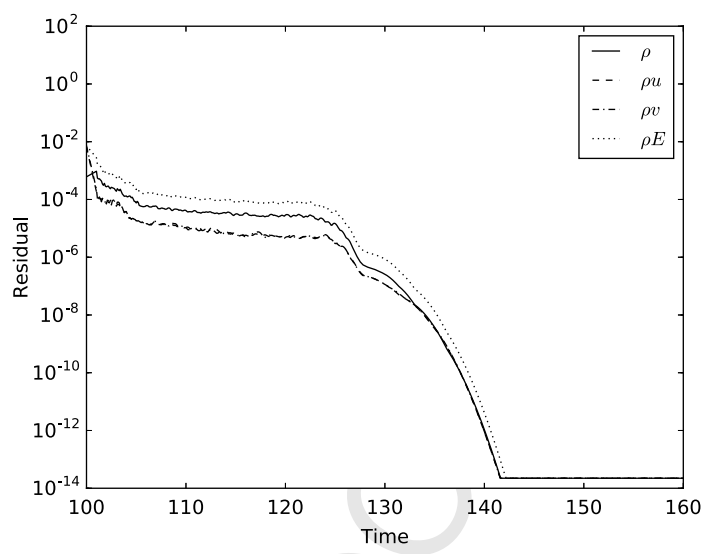

(b) Implicit; Backward Euler

Fig. 5. Example 1 - Residual history for a) third-order strong stability preserving (SSP) Runge-Kutta time scheme, and b) Backward Euler time scheme.

Table 1

Verification - Order of accuracy verification for the second-order DG with and without WENO on irregular triangular elements.

\begin{tabular}{|c|c|c|c|c|c|c|c|c|c|}
\hline \multirow[t]{2}{*}{ Grid } & \multirow[t]{2}{*}{$h$ size } & \multicolumn{4}{|c|}{$L_{2}$ Error: DG $(P 1)$} & \multicolumn{4}{|c|}{ Order: DG (P1) } \\
\hline & & $\rho$ & $\rho u$ & $\rho v$ & $\rho E$ & $\rho$ & $\rho u$ & $\rho v$ & $\rho E$ \\
\hline $20 \times 20$ & $2.66 \mathrm{E}-1$ & $6.015 \mathrm{E}-3$ & $6.323 \mathrm{E}-3$ & $6.328 \mathrm{E}-3$ & $2.056 \mathrm{E}-2$ & - & - & - & - \\
\hline $40 \times 40$ & $1.40 \mathrm{E}-1$ & $1.452 \mathrm{E}-3$ & $1.520 \mathrm{E}-3$ & $1.524 \mathrm{E}-3$ & $4.960 \mathrm{E}-3$ & 2.21 & 2.22 & 2.21 & 2.22 \\
\hline $60 \times 60$ & 8.93E-2 & $5.950 \mathrm{E}-4$ & $6.279 \mathrm{E}-4$ & $6.270 \mathrm{E}-4$ & $2.056 \mathrm{E}-3$ & 1.99 & 1.97 & 1.98 & 1.96 \\
\hline $80 \times 80$ & $6.93 \mathrm{E}-2$ & $3.422 \mathrm{E}-4$ & $3.602 \mathrm{E}-4$ & $3.595 \mathrm{E}-4$ & $1.176 \mathrm{E}-3$ & 2.18 & 2.19 & 2.19 & 2.20 \\
\hline $100 \times 100$ & $5.61 \mathrm{E}-2$ & $2.170 \mathrm{E}-4$ & $2.255 \mathrm{E}-4$ & $2.260 \mathrm{E}-4$ & $7.449 \mathrm{E}-4$ & 2.17 & 2.23 & 2.21 & 2.17 \\
\hline \multirow[t]{2}{*}{ Grid } & \multirow[t]{2}{*}{$h$ size } & \multicolumn{4}{|c|}{$L_{2}$ Error: WENO-DG $(P 1)$} & \multicolumn{4}{|c|}{ Order: WENO-DG $(P 1)$} \\
\hline & & $\rho$ & $\rho u$ & $\rho v$ & $\rho E$ & $\rho$ & $\rho u$ & $\rho v$ & $\rho E$ \\
\hline $20 \times 20$ & $2.66 \mathrm{E}-1$ & $6.015 \mathrm{E}-3$ & $6.323 \mathrm{E}-3$ & $6.328 \mathrm{E}-3$ & $2.056 \mathrm{E}-2$ & - & - & - & - \\
\hline $40 \times 40$ & $1.40 \mathrm{E}-1$ & $1.452 \mathrm{E}-3$ & $1.520 \mathrm{E}-3$ & $1.524 \mathrm{E}-3$ & $4.960 \mathrm{E}-3$ & 2.21 & 2.22 & 2.21 & 2.22 \\
\hline $60 \times 60$ & $8.93 E-2$ & $5.950 \mathrm{E}-4$ & $6.279 \mathrm{E}-4$ & $6.270 \mathrm{E}-4$ & $2.056 \mathrm{E}-3$ & 1.99 & 1.97 & 1.98 & 1.96 \\
\hline $80 \times 80$ & $6.93 \mathrm{E}-2$ & $3.422 \mathrm{E}-4$ & $3.602 \mathrm{E}-4$ & $3.595 \mathrm{E}-4$ & $1.176 \mathrm{E}-3$ & 2.18 & 2.19 & 2.19 & 2.20 \\
\hline $100 \times 100$ & $5.61 \mathrm{E}-2$ & $2.170 \mathrm{E}-4$ & $2.255 \mathrm{E}-4$ & $2.260 \mathrm{E}-4$ & $7.449 \mathrm{E}-4$ & 2.17 & 2.23 & 2.21 & 2.17 \\
\hline
\end{tabular}

\subsection{Polynomial reconstruction approach 2}

In this section, a second approach in constructing the polynomials $\tilde{p}^{(i)}$ is presented. In this approach, a constraint minimization problem is solved using the least squares $(l s q)$ approach. The proposed $l s q$ linear polynomial reconstruction procedure for unstructured DG schemes is also compact, and its implementation is nonintrusive similar to the first presented approach.

The aim here is to construct a set of linear polynomials, $\tilde{p}^{l s q}$, on the target cell such that

$$
\arg \min _{\tilde{u}} \sum_{l \in S}\left(\frac{1}{\left|\Delta_{l}\right|} \int_{\Delta_{l}} \tilde{p}^{l s q}(\tilde{u}, x) d \mathbf{x}-\bar{u}^{(l)}\right)^{2}, \quad S=\{1,2,3\},
$$

where $l$ corresponds to the immediate neighbors of the target element, is satisfied subject to a constraint that the means of the constructed polynomials remain the same as the means of the original polynomials on the target element; i.e.,

$$
\int_{\Delta_{0}} \tilde{p}^{l s q} d \mathbf{x}=\bar{u}^{(0)}
$$

Thus, the following minimization problem is sought by employing a penalization parameter (called Lagrange multiplier) $\lambda$ :

$$
E\left(\tilde{u}_{i}, \lambda\right)=\arg \min _{\tilde{u}_{i}}\left[\sum_{l \in S}\left(\frac{1}{\left|\Delta_{l}\right|} \int_{\Delta_{l}} \tilde{p}^{l s q}\left(\tilde{u}_{i}, x\right) d \mathbf{x}-\bar{u}^{(l)}\right)^{2}+\lambda\left(\frac{1}{\left|\Delta_{0}\right|} \int_{\Delta_{0}} \tilde{p}^{l s q}\left(\tilde{u}_{i}, x\right) d \mathbf{x}-\bar{u}^{(0)}\right)\right],
$$


Table 2

Verification - Order of accuracy verification for the third-order DG with and without WENO on irregular triangular elements.

\begin{tabular}{|c|c|c|c|c|c|c|c|c|c|}
\hline \multirow[t]{2}{*}{ Grid } & \multirow[t]{2}{*}{$h$ size } & \multicolumn{4}{|c|}{$L_{2}$ Error: DG $(P 2)$} & \multicolumn{4}{|c|}{ Order: DG $(P 2)$} \\
\hline & & $\rho$ & $\rho u$ & $\rho v$ & $\rho E$ & $\rho$ & $\rho u$ & $\rho v$ & $\rho E$ \\
\hline $20 \times 20$ & $2.66 \mathrm{E}-1$ & $3.679 \mathrm{E}-4$ & $2.752 \mathrm{E}-4$ & $2.795 \mathrm{E}-4$ & $1.153 \mathrm{E}-3$ & - & - & - & - \\
\hline $40 \times 40$ & $1.40 \mathrm{E}-1$ & $4.803 \mathrm{E}-5$ & $3.425 \mathrm{E}-5$ & $3.455 \mathrm{E}-5$ & $1.497 \mathrm{E}-4$ & 3.17 & 3.34 & 3.25 & 3.18 \\
\hline $60 \times 60$ & $8.93 \mathrm{E}-2$ & $1.438 \mathrm{E}-5$ & $9.911 \mathrm{E}-6$ & $9.873 \mathrm{E}-6$ & $4.455 \mathrm{E}-5$ & 2.69 & 2.76 & 2.79 & 2.70 \\
\hline $80 \times 80$ & $6.93 \mathrm{E}-2$ & 6.079E-6 & 4.237E-6 & $4.238 \mathrm{E}-6$ & $1.885 \mathrm{E}-5$ & 3.39 & 3.35 & 3.34 & 3.39 \\
\hline $100 \times 100$ & $5.61 \mathrm{E}-2$ & $3.123 E-6$ & $2.114 \mathrm{E}-6$ & $2.120 \mathrm{E}-6$ & $9.690 \mathrm{E}-6$ & 3.17 & 3.31 & 3.29 & 3.16 \\
\hline \multirow[t]{2}{*}{ Grid } & \multirow[t]{2}{*}{$h$ size } & \multicolumn{4}{|c|}{$L_{2}$ Error: WENO-DG $(P 2)$} & \multicolumn{4}{|c|}{ Order: WENO-DG (P2) } \\
\hline & & $\rho$ & $\rho u$ & $\rho v$ & $\rho E$ & $\bar{\rho}$ & $\rho u$ & $\rho v$ & $\rho E$ \\
\hline $20 \times 20$ & $2.66 \mathrm{E}-1$ & 3.679E-4 & $2.752 \mathrm{E}-4$ & $2.795 \mathrm{E}-4$ & $1.153 \mathrm{E}-3$ & - & - & - & - \\
\hline $40 \times 40$ & $1.40 \mathrm{E}-1$ & $4.803 \mathrm{E}-5$ & $3.425 \mathrm{E}-5$ & $3.455 \mathrm{E}-5$ & $1.497 \mathrm{E}-4$ & 3.17 & 3.34 & 3.25 & 3.18 \\
\hline $60 \times 60$ & $8.93 \mathrm{E}-2$ & $1.438 \mathrm{E}-5$ & $9.911 \mathrm{E}-6$ & $9.873 \mathrm{E}-6$ & $4.455 \mathrm{E}-5$ & 2.69 & 2.76 & 2.79 & 2.70 \\
\hline $80 \times 80$ & $6.93 \mathrm{E}-2$ & $6.079 \mathrm{E}-6$ & $4.237 \mathrm{E}-6$ & $4.238 \mathrm{E}-6$ & $1.885 \mathrm{E}-5$ & 3.39 & 3.35 & 3.34 & 3.39 \\
\hline $100 \times 100$ & $5.61 \mathrm{E}-2$ & $3.123 \mathrm{E}-6$ & $2.114 \mathrm{E}-6$ & $2.120 \mathrm{E}-6$ & $9.690 \mathrm{E}-6$ & 3.17 & 3.31 & 3.29 & 3.16 \\
\hline
\end{tabular}

Table 3

Verification - Order of accuracy verification for the fourth-order DG with and without WENO on irregular triangular elements.

\begin{tabular}{|c|c|c|c|c|c|c|c|c|c|}
\hline \multirow[t]{2}{*}{ Grid } & \multirow[t]{2}{*}{$h$ size } & \multicolumn{4}{|c|}{$L_{2}$ Error: DG $(P 3)$} & \multicolumn{4}{|c|}{ Order: DG (P3) } \\
\hline & & $\rho$ & $\rho u$ & $\rho v$ & $\rho E$ & $\rho$ & $\rho u$ & $\rho v$ & $\rho E$ \\
\hline $20 \times 20$ & $2.66 \mathrm{E}-1$ & 7.134E-6 & 7.199E-6 & $7.190 \mathrm{E}-6$ & $2.358 \mathrm{E}-5$ & - & - & - & - \\
\hline $40 \times 40$ & $1.40 \mathrm{E}-1$ & $4.448 \mathrm{E}-7$ & 4.377E-7 & $4.385 \mathrm{E}-7$ & $1.459 \mathrm{E}-6$ & 4.32 & 4.36 & 4.35 & 4.33 \\
\hline $60 \times 60$ & $8.93 \mathrm{E}-2$ & $7.258 \mathrm{E}-8$ & $7.195 \mathrm{E}-8$ & $7.213 E-8$ & $2.418 \mathrm{E}-7$ & 4.04 & 4.02 & 4.02 & 4.00 \\
\hline $80 \times 80$ & $6.93 \mathrm{E}-2$ & $2.374 \mathrm{E}-8$ & $2.373 \mathrm{E}-8$ & $2.365 \mathrm{E}-8$ & $7.874 \mathrm{E}-8$ & 4.41 & 4.37 & 4.40 & 4.42 \\
\hline $100 \times 100$ & $5.61 \mathrm{E}-2$ & $9.509 \mathrm{E}-9$ & $9.330 \mathrm{E}-9$ & $9.303 \mathrm{E}-9$ & $3.145 \mathrm{E}-8$ & 4.35 & 4.44 & 4.44 & 4.36 \\
\hline \multirow[t]{2}{*}{ Grid } & \multirow[t]{2}{*}{$h$ size } & \multicolumn{4}{|c|}{$L_{2}$ Error: WENO-DG $(P 3)$} & \multicolumn{4}{|c|}{ Order: WENO-DG (P3) } \\
\hline & & $\rho$ & $\rho u$ & $\rho v$ & $\rho E$ & $\rho$ & $\rho u$ & $\rho v$ & $\rho E$ \\
\hline $20 \times 20$ & $2.66 \mathrm{E}-1$ & 7.176E-6 & 7.212E-6 & $7.221 \mathrm{E}-6$ & $2.371 \mathrm{E}-5$ & - & - & - & - \\
\hline $40 \times 40$ & $1.40 \mathrm{E}-1$ & $4.426 \mathrm{E}-7$ & 4.369E-7 & $4.374 \mathrm{E}-7$ & $1.455 \mathrm{E}-6$ & 4.33 & 4.36 & 4.35 & 4.34 \\
\hline $60 \times 60$ & 8.93E-2 & $7.273 \mathrm{E}-8$ & $7.206 \mathrm{E}-8$ & $7.227 \mathrm{E}-8$ & $2.423 \mathrm{E}-7$ & 4.02 & 4.01 & 4.01 & 3.99 \\
\hline $80 \times 80$ & $6.93 \mathrm{E}-2$ & $2.373 \mathrm{E}-8$ & $2.374 \mathrm{E}-8$ & $2.367 \mathrm{E}-8$ & $7.880 \mathrm{E}-8$ & 4.42 & 4.38 & 4.40 & 4.43 \\
\hline $100 \times 100$ & $5.61 \mathrm{E}-2$ & $9.455 \mathrm{E}-9$ & $9.326 \mathrm{E}-9$ & $9.301 \mathrm{E}-9$ & 3.137E-8 & 4.37 & 4.44 & 4.44 & 4.38 \\
\hline
\end{tabular}

Table 4

Verification - Order of accuracy verification for the fifth-order DG with and without WENO on irregular triangular elements.

\begin{tabular}{|c|c|c|c|c|c|c|c|c|c|}
\hline \multirow[t]{2}{*}{ Grid } & \multirow[t]{2}{*}{$h$ size } & \multicolumn{4}{|c|}{$L_{2}$ Error: DG $(P 4)$} & \multicolumn{4}{|c|}{ Order: DG (P4) } \\
\hline & & $\rho$ & $\rho u$ & $\rho v$ & $\rho E$ & $\rho$ & $\rho u$ & $\rho v$ & $\rho E$ \\
\hline $20 \times 20$ & $2.66 \mathrm{E}-1$ & $2.285 \mathrm{E}-7$ & $1.849 \mathrm{E}-7$ & $1.879 \mathrm{E}-7$ & $7.260 \mathrm{E}-7$ & - & - & - & - \\
\hline $40 \times 40$ & $1.40 \mathrm{E}-1$ & $7.790 \mathrm{E}-9$ & $5.853 \mathrm{E}-9$ & $5.993 \mathrm{E}-7$ & $2.455 \mathrm{E}-8$ & 5.26 & 5.37 & 5.36 & 5.27 \\
\hline $60 \times 60$ & $8.93 \mathrm{E}-2$ & $9.745 \mathrm{E}-10$ & 7.319E-10 & $7.275 \mathrm{E}-10$ & $3.050 \mathrm{E}-9$ & 4.63 & 4.63 & 4.70 & 4.65 \\
\hline $80 \times 80$ & $6.93 \mathrm{E}-2$ & $1.362 \mathrm{E}-10$ & $1.779 \mathrm{E}-10$ & $1.766 \mathrm{E}-10$ & $7.412 \mathrm{E}-10$ & 5.59 & 5.58 & 5.58 & 5.58 \\
\hline \multirow[t]{2}{*}{ Grid } & \multirow[t]{2}{*}{$h$ size } & \multicolumn{4}{|c|}{$L_{2}$ Error: WENO-DG $(P 4)$} & \multicolumn{4}{|c|}{ Order: WENO-DG (P4) } \\
\hline & & $\rho$ & $\rho u$ & $\rho v$ & $\rho E$ & $\rho$ & $\rho u$ & $\rho v$ & $\rho E$ \\
\hline $20 \times 20$ & $2.66 \mathrm{E}-1$ & $2.285 \mathrm{E}-7$ & $1.849 \mathrm{E}-7$ & $1.879 \mathrm{E}-7$ & $7.260 \mathrm{E}-7$ & - & - & - & - \\
\hline $40 \times 40$ & $1.40 \mathrm{E}-1$ & $7.890 \mathrm{E}-9$ & $5.853 \mathrm{E}-9$ & $5.996 \mathrm{E}-7$ & $2.455 \mathrm{E}-8$ & 5.26 & 5.37 & 5.36 & 5.27 \\
\hline $60 \times 60$ & 8.93E-2 & $1.034 \mathrm{E}-9$ & $7.604 \mathrm{E}-10$ & $7.532 \mathrm{E}-10$ & $3.185 \mathrm{E}-9$ & 4.50 & 4.55 & 4.62 & 4.55 \\
\hline $80 \times 80$ & $6.93 \mathrm{E}-2$ & $2.363 \mathrm{E}-10$ & $1.780 \mathrm{E}-10$ & $1.767 \mathrm{E}-10$ & $7.413 \mathrm{E}-10$ & 5.82 & 5.73 & 5.73 & 5.75 \\
\hline
\end{tabular}



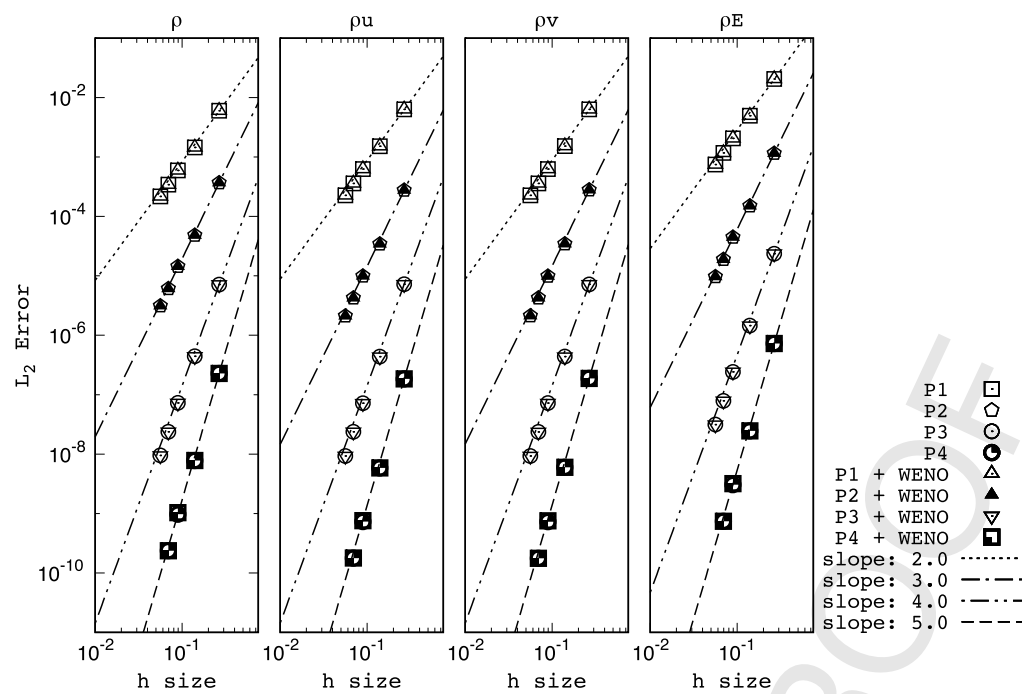

Fig. 6. Verification - Order of accuracy plots for the conserved density, x and y momentums, and energy for DG and the proposed WENO-DG on irregular triangular elements.
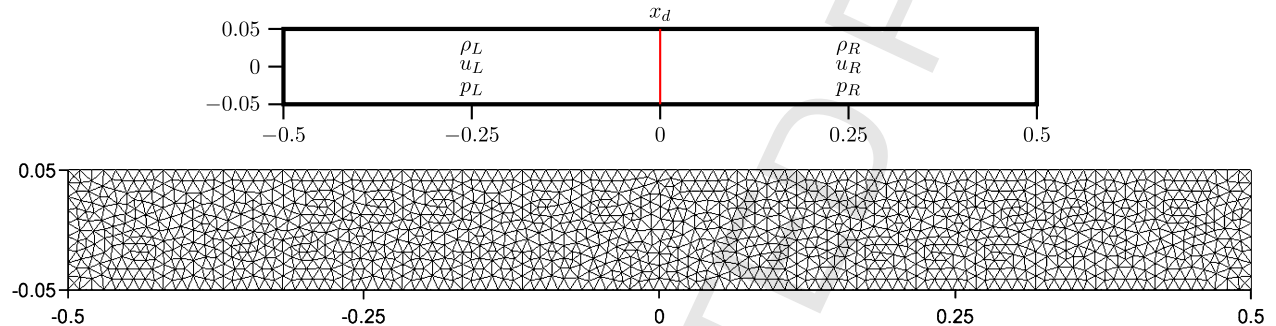

Fig. 7. Schematic of the Riemann problem, and the irregular triangular grid with $h=1 / 100$. The two states of the gas are separated at $x_{d}=0$.

Table 5

Initial left (L) and right (R) states used for the Sod and Lax test cases. The position of the initial discontinuity, $x_{d}$ and final simulation times, $t_{\text {final }}$ are also given. See Fig. 7 for geometry information.

\begin{tabular}{llllllll}
\hline Test case & $\rho_{L}$ & $u_{L}$ & $p_{L}$ & $\rho_{R}$ & $u_{R}$ & $p_{R}$ \\
\hline Sod & 1.0 & 0.0 & 1.0 & 0.125 & 0.0 & 0.1 \\
Lax & 0.445 & 0.698 & 3.528 & 0.5 & 0.0 & 0.0 & 0.571 \\
\hline
\end{tabular}

where $\tilde{u}_{i}$ are the coefficients of the to-be-constructed linear polynomial for $i$ th degrees of freedom, dof, and $\left|\Delta_{l}\right|$ is the volume of the neighbor $l$. The constraint minimization problem may be expanded and expressed with employing proper quadrature rules as

$$
\begin{aligned}
& E\left(\tilde{u}_{i}, \lambda\right)= \\
& \arg \min _{\tilde{u}_{i}}\left[\sum_{l \in S}\left(\frac{1}{\left|\Delta_{l}\right|} \sum_{i} \sum_{q} \tilde{u}_{i} \tilde{\varphi}_{i, q}^{(0 \rightarrow l)} \omega_{q}^{(l)}|\operatorname{det} J|^{(l)}-\bar{u}^{(l)}\right)^{2}+\lambda\left(\frac{1}{\left|\Delta_{0}\right|} \sum_{i} \sum_{q} \tilde{u}_{i} \tilde{\varphi}_{i, q}^{(0)} \omega_{q}^{(0)}|\operatorname{det} J|^{(0)}-\bar{u}^{(0)}\right)\right],
\end{aligned}
$$

where $\omega_{q}^{(l)}$ is the weight of the quadrature for the qth quadrature point of the lth neighbor, $|\operatorname{det} J|^{(l)}=\left|\Delta_{0}\right|^{(l)}$ is the determinant of the Jacobian of the transformation from the reference to the physical element of neighbor $l$, and $\varphi_{i, q}^{(0 \rightarrow l)}$ is the $i$ th basis function in the reference element of the target element extrapolated to the lth neighbor, and evaluated at the corresponding qth quadrature point.

The minimization problem is solved by setting the derivatives of $E$ w.r.t. the $j$ th dof coefficient $\tilde{u}_{j}$ and $\lambda$ to zero; i.e.,

$$
\frac{\partial E}{\partial \tilde{u}_{j}}=\sum_{l}\left(\frac{1}{\left|\Delta_{l}\right|} \sum_{q} \tilde{\varphi}_{j, q}^{(0 \rightarrow l)} \omega_{q}^{(l)}|\operatorname{det} J|^{(l)}\right)\left(\frac{1}{\left|\Delta_{l}\right|} \sum_{i} \sum_{q} \tilde{u}_{i} \tilde{\varphi}_{i, q}^{(0 \rightarrow l)} \omega_{q}^{(l)}|\operatorname{det} J|^{(l)}-\bar{u}^{(l)}\right)
$$




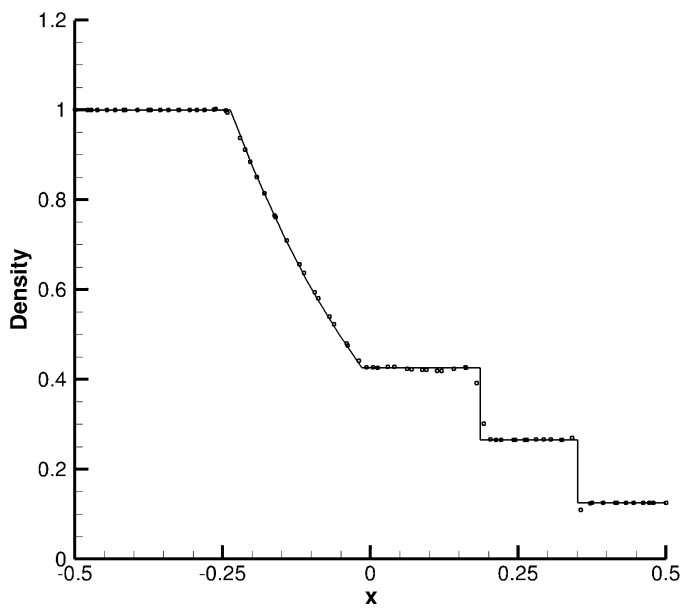

(a) second-order

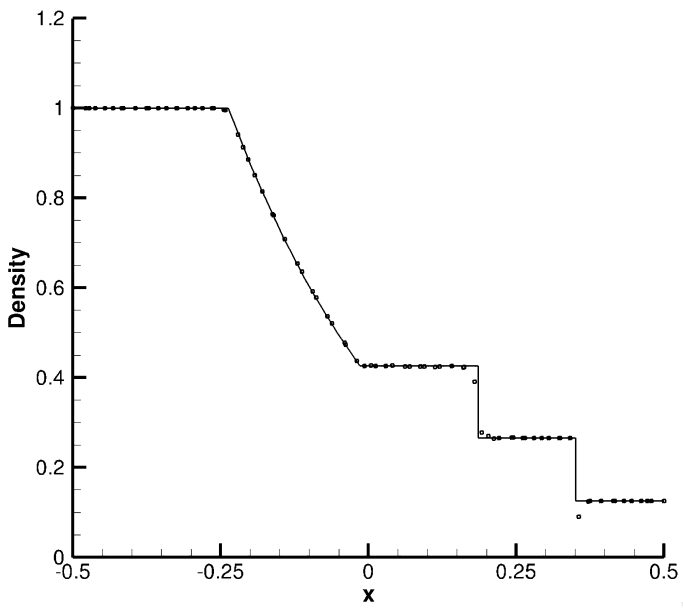

(c) fourth-order

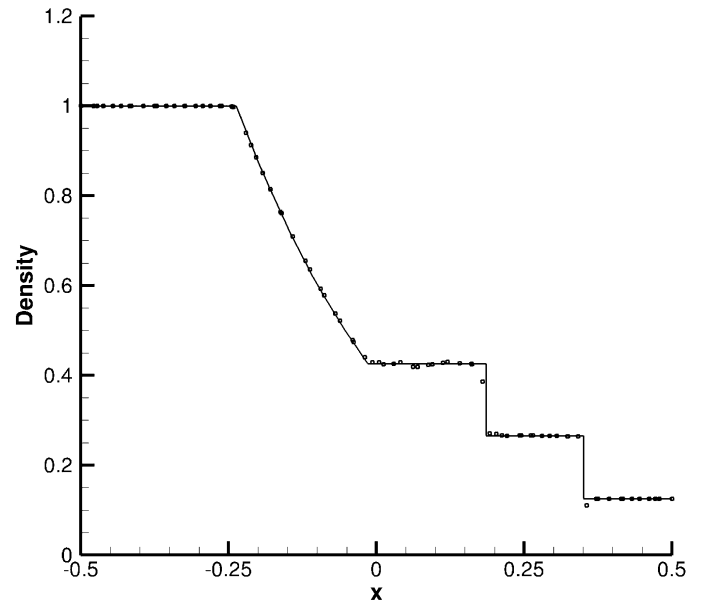

(b) third-order

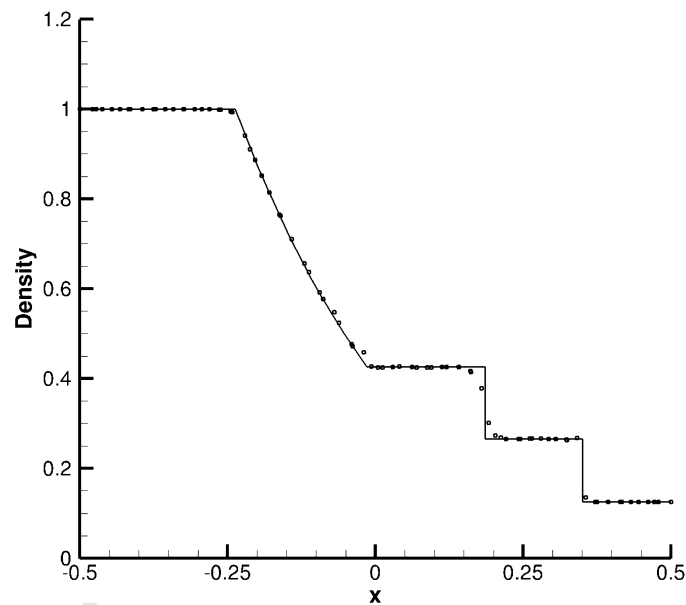

(d) fifth-order

Fig. 8. Predicted density profile for the Sod problem with $h / 100$ irregular triangular elements at $t=0.2$, and comparison against the exact solution.

$$
\begin{gathered}
+\tilde{\lambda}\left(\frac{1}{\left|\Delta_{0}\right|} \sum_{q} \tilde{\varphi}_{j, q}^{(0)} \omega_{q}^{(0)}|\operatorname{det} J|^{(0)}\right)=0, \\
\frac{\partial E}{\partial \lambda}=\frac{1}{\left|\Delta_{0}\right|} \sum_{i} \sum_{q} \tilde{u}_{i} \tilde{\varphi}_{i, q}^{(0)} \omega_{q}^{(0)}|\operatorname{det} J|^{(0)}-\bar{u}^{(0)}=0 .
\end{gathered}
$$

This may be expressed in a matrix form as

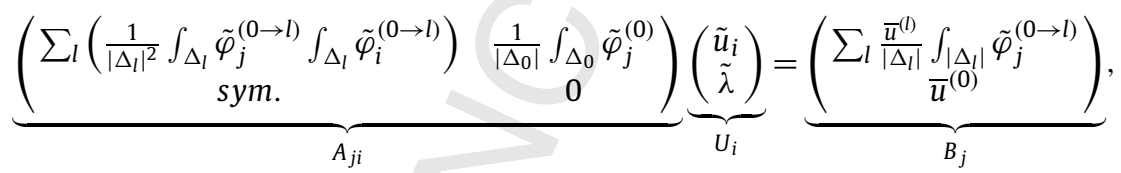

where $A_{j i}$ is a symmetric matrix, and $\tilde{\lambda}=\lambda / 2$.

It is important to note that whenever the integration is performed on the neighbor element $l$, the linear polynomial basis defined on the target element, $\tilde{\varphi}^{(0)}$, must be extrapolated to the neighboring element. This is emphasized in Eq. (24) by employing the notation $\varphi_{j}^{(0 \rightarrow l)}$. For convenience and simplicity in the discussion, however, this notation is removed in the subsequent text, and such extrapolation is therefore assumed to be understood whenever the integration is performed on the neighboring elements.

For interior triangular elements similar to one shown in Fig. 1a, the matrix $A_{j i}$ is a symmetric $4 \times 4$ matrix, and $U_{i}$ and $B_{j}$ are vectors of length four (three for $d o f+$ one for the constraint). They take the following forms 


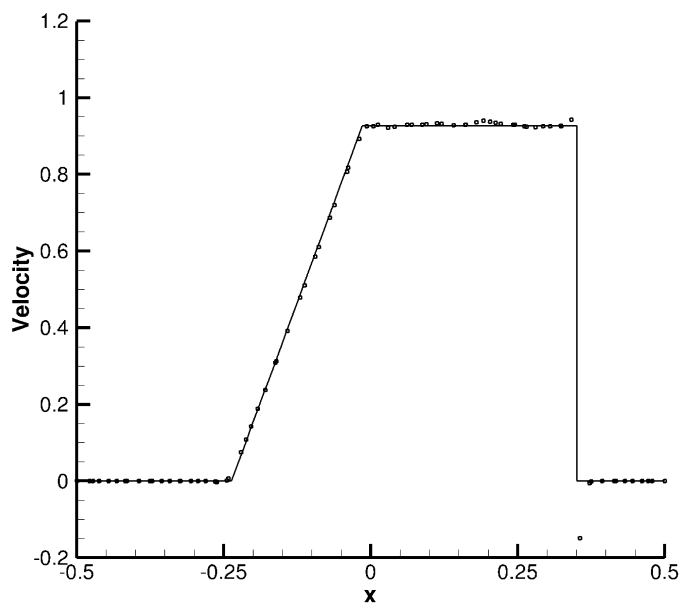

(a) second-order

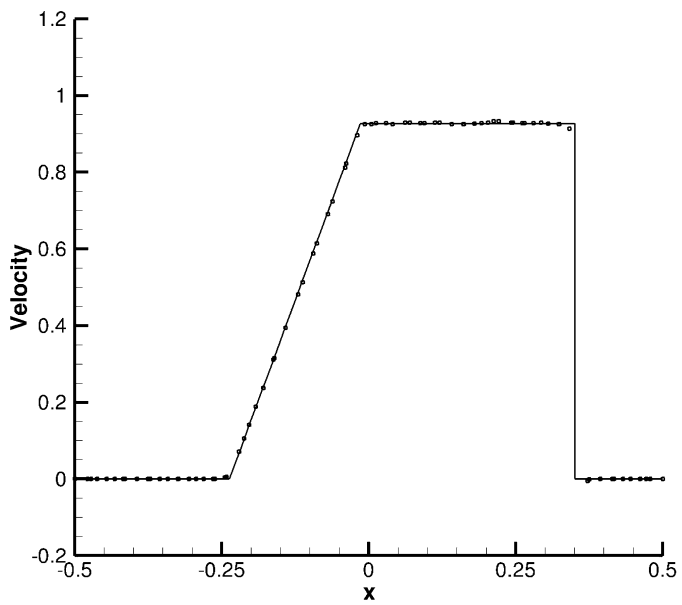

(c) fourth-order

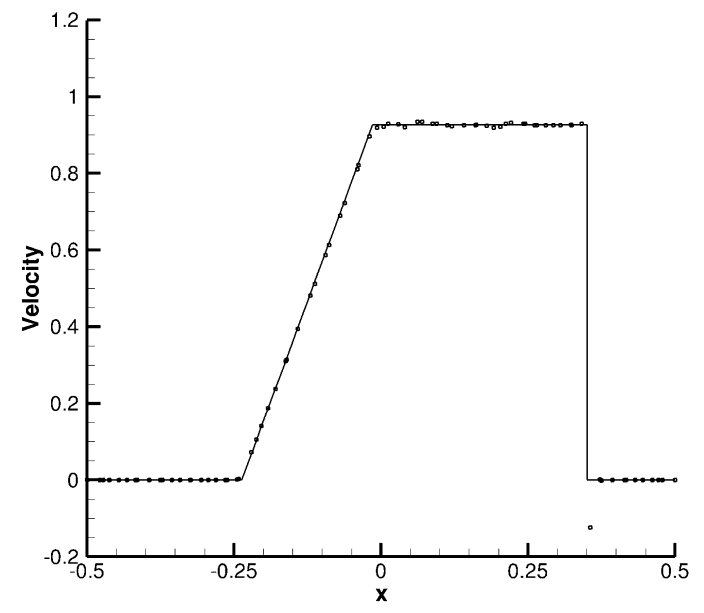

(b) third-order

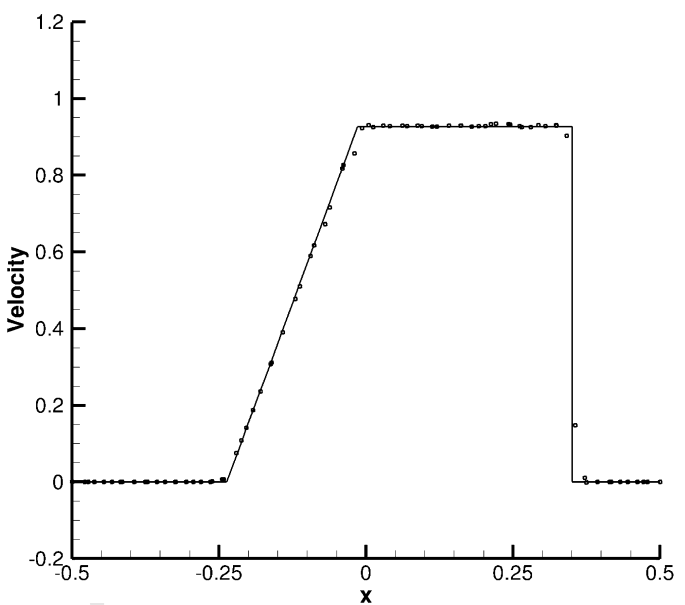

(d) fifth-order

Fig. 9. Predicted velocity profile for the Sod problem with $h / 100$ irregular triangular elements at $t=0.2$, and comparison against the exact solution.

$$
A_{j i}=\left(\begin{array}{cccc}
\sum_{l} \frac{1}{\left|\Delta_{l}\right|^{2}}\left(\int_{\Delta_{l}} \tilde{\varphi}_{0}^{(0)}\right)^{2} & \sum_{l} \frac{1}{\left|\Delta_{l}\right|^{2}} \int_{\Delta_{l}} \tilde{\varphi}_{0}^{(0)} \int_{\left|\Delta_{l}\right|} \tilde{\varphi}_{1}^{(0)} & \sum_{l} \frac{1}{\left|\Delta_{l}\right|^{2}} \int_{\Delta_{l}} \tilde{\varphi}_{0}^{(0)} \int_{\mid \Delta_{l}} \tilde{\varphi}_{2}^{(0)} & \frac{1}{\left|\Delta_{0}\right|} \int_{\Delta_{0}} \tilde{\varphi}_{0}^{(0)} \\
\text { Sym. } & \sum_{l} \frac{1}{\left|\Delta_{l}\right|^{2}}\left(\int_{\Delta_{l}} \tilde{\varphi}_{1}^{(0)}\right)^{2} & \sum_{l} \frac{1}{\left|\Delta_{l}\right|^{2}} \int_{\Delta_{l}} \tilde{\varphi}_{1}^{(0)} \int_{\Delta_{l}} \tilde{\varphi}_{2}^{(0)} & \frac{1}{\left|\Delta_{0}\right|} \int_{\Delta_{0}} \tilde{\varphi}_{1}^{(0)} \\
\text { Sym. } & \text { Sym. } & \sum_{l} \frac{1}{\left|\Delta_{l}\right|^{2}}\left(\int_{\Delta_{l}} \tilde{\varphi}_{2}^{(0)}\right)^{2} & \frac{1}{\left|\Delta_{0}\right|} \int_{\Delta_{0}} \tilde{\varphi}_{2}^{(0)} \\
\text { Sym. } & \text { Sym. } & \text { Sym. } & 0
\end{array}\right) \text {, }
$$

$$
U_{i}=\left(\begin{array}{c}
\tilde{u}_{0} \\
\tilde{u}_{1} \\
\tilde{u}_{2} \\
\tilde{\lambda}
\end{array}\right), \quad B_{j}=\left(\begin{array}{c}
\sum_{l} \frac{\bar{u}^{(l)}}{\mid \Delta_{l}} \int_{\Delta_{l}} \tilde{\varphi}_{0}^{(0)} \\
\sum_{l} \frac{\bar{u}^{(l)}}{\mid \Delta_{l}} \int_{\Delta_{l}} \tilde{\varphi}_{1}^{(0)} \\
\sum_{l} \frac{\bar{u}^{(l)}}{\left|\Delta_{l}\right|} \int_{\Delta_{l}} \tilde{\varphi}_{2}^{(0)} \\
\bar{u}^{(0)}
\end{array}\right) .
$$

For triangular elements whose faces may be on boundaries, such as the one depicted in Fig. $1 \mathrm{~b}$, where two of the target element faces are on the boundary, the set of neighbors in the linear reconstruction is therefore $l=\left\{1, \partial \Omega_{1}, \partial \Omega_{2}\right\}$, where $\partial \Omega$ denotes the corresponding boundary face of the target element that is on a boundary. For instance, consider the triangle shown in Fig. 1b, for which the following expressions for the $A_{11}$ and its corresponding right-hand-side $B_{1}$ are obtained

$$
A_{11}=\frac{1}{\left|\Delta_{1}\right|^{2}}\left(\int_{\Delta_{1}} \tilde{\varphi}_{0}^{(0)}\right)^{2}+\frac{1}{\left|\partial \Omega_{1}\right|^{2}}\left(\int_{\partial \Omega_{1}} \tilde{\varphi}_{0}^{(0)}\right)^{2}+\frac{1}{\left|\partial \Omega_{2}\right|^{2}}\left(\int_{\partial \Omega_{2}} \tilde{\varphi}_{0}^{(0)}\right)^{2},
$$




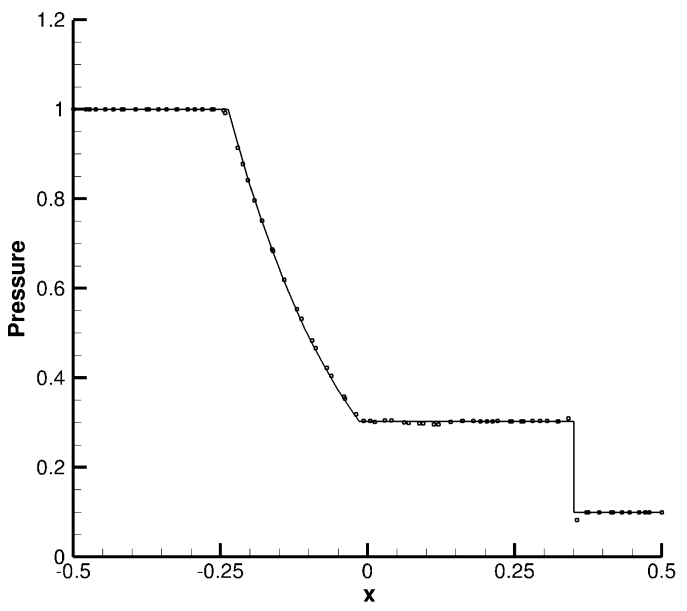

(a) second-order

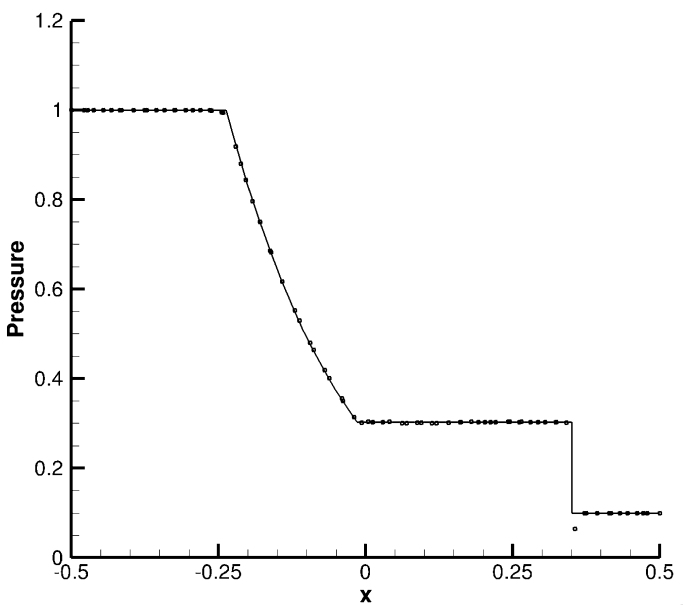

(c) fourth-order

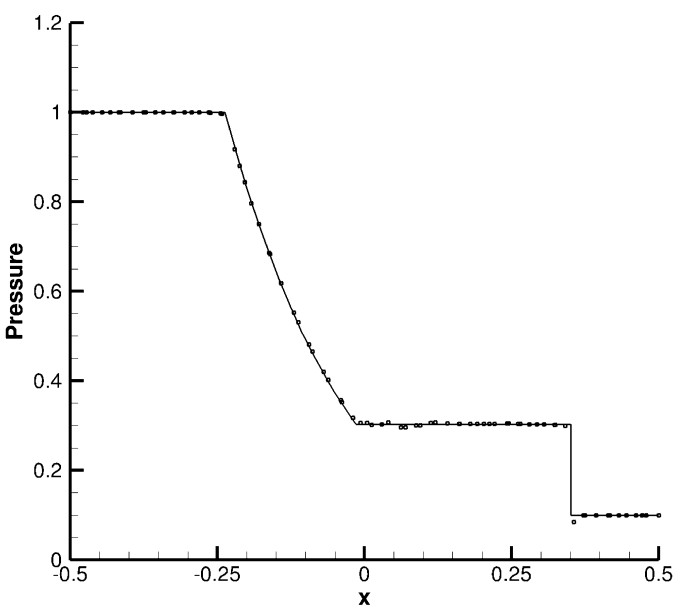

(b) third-order

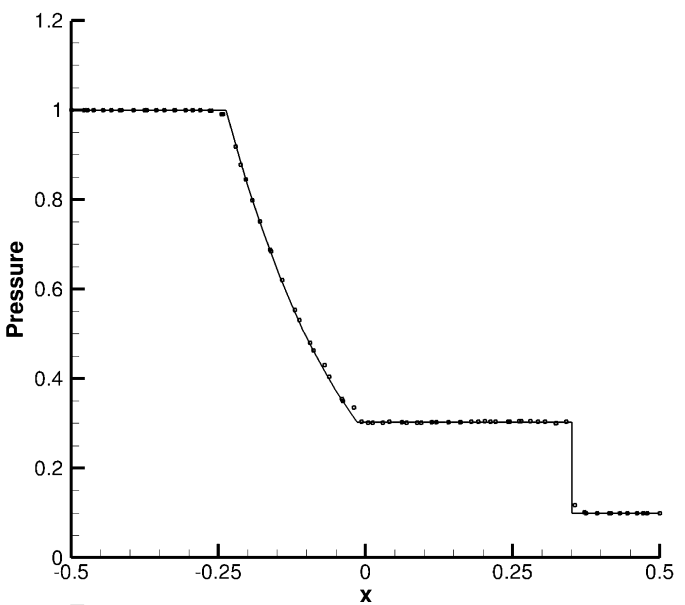

(d) fifth-order

Fig. 10. Predicted pressure profile for the Sod problem with $h / 100$ irregular triangular elements at $t=0.2$, and comparison against the exact solution.

$$
B_{1}=\frac{\bar{u}^{(1)}}{\left|\Delta_{1}\right|} \int_{\left|\Delta_{1}\right|} \tilde{\varphi}_{0}^{(0)}+\frac{\bar{u}_{\partial \Omega_{1}}}{\left|\partial \Omega_{1}\right|} \int_{\partial \Omega_{1}} \tilde{\varphi}_{0}^{(0)}+\frac{\bar{u}_{\partial \Omega_{2}}}{\left|\partial \Omega_{2}\right|} \int_{\partial \Omega_{2}} \tilde{\varphi}_{0}^{(0)},
$$

where $\bar{u}_{\partial \Omega_{l}}$ is the mean value evaluated on the corresponding boundary face of the target element after the boundary condition is applied. Note that $\partial \Omega_{l}$ denotes that the integration is performed on the corresponding boundary face of the target element and thus, no extrapolation is needed for the last two terms of the expression (27); extrapolation must be applied to the first term of the expression.

The complete step-by-step procedure with the second presented approach is outlined below.

\subsubsection{Scalar case}

1. Compute the means of the polynomials on the compact WENO stencil $\{0,1,2,3\}$. This is the same as the step 1 of the approach 1.

2. Construct the $\tilde{p}^{l s q}$ polynomial as outlined above.

3. Use $\tilde{p}^{l s q}$, and follow steps 3 and 4 of approach 1 , and evaluate $\tilde{p}^{(0)^{\text {new }}}$; i.e.,

$$
p^{(0)^{n e w}}=\frac{\omega_{0}}{\gamma_{0}} p^{(0)}+\left(\omega^{l s q}-\omega_{0} \frac{\gamma^{l s q}}{\gamma_{0}}\right) \tilde{p}^{l s q} .
$$

\subsubsection{System case}

1. Follow steps 1 and 2 given for the scaler case. 


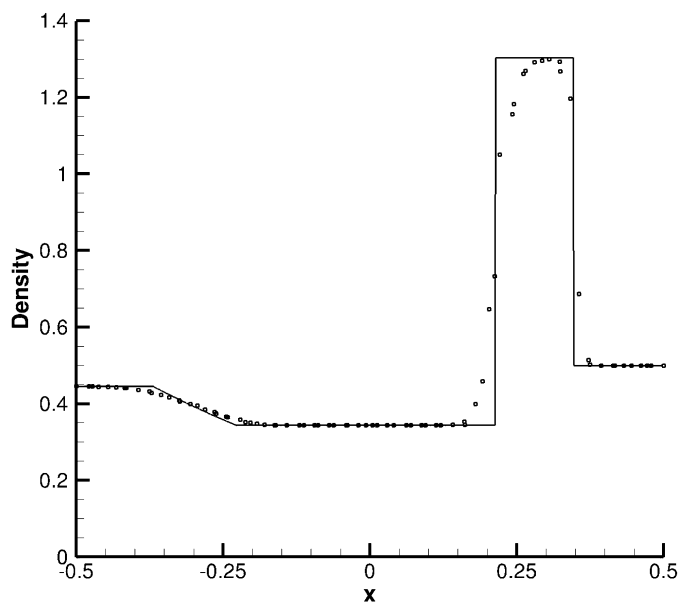

(a) second-order

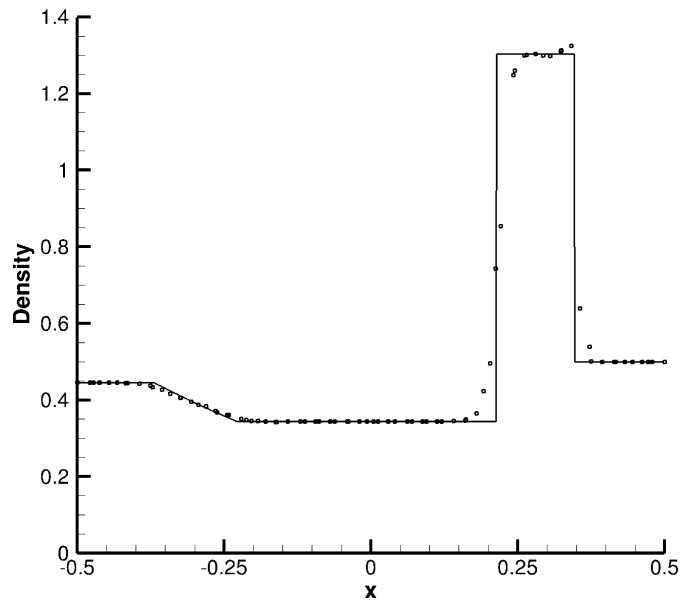

(c) fourth-order

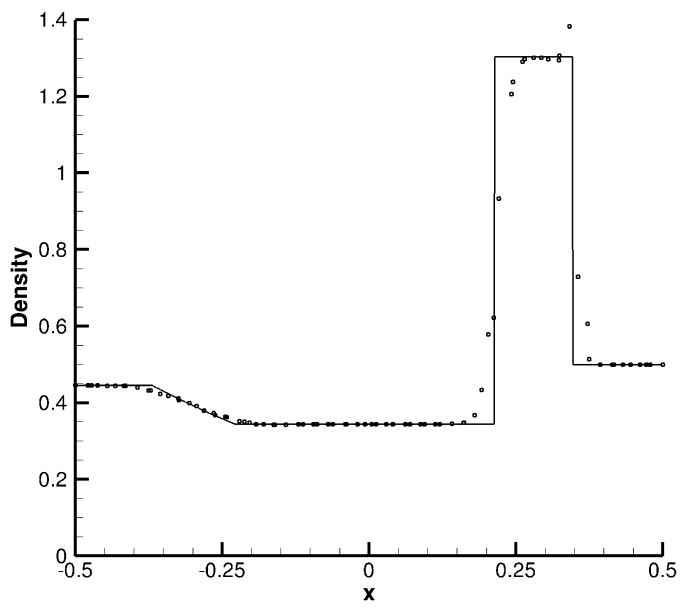

(b) third-order

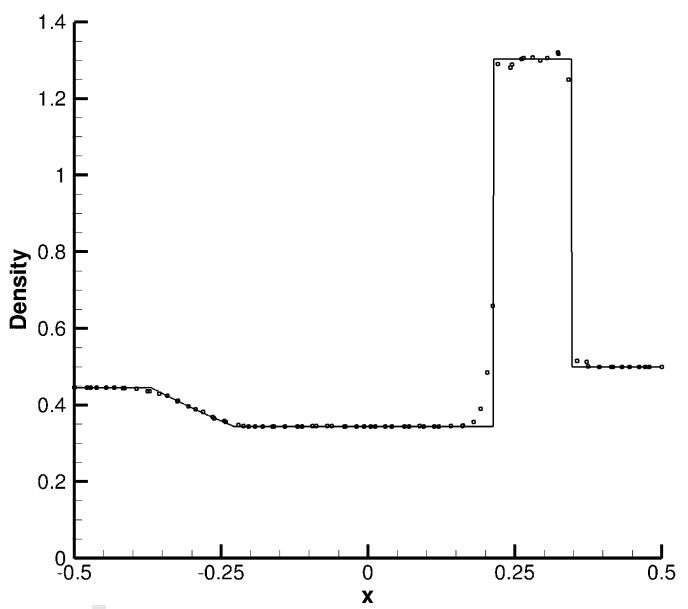

(d) fifth-order

Fig. 11. Predicted density profile for the Lax problem with $h / 100$ irregular triangular elements at $t=0.14$, and comparison against the exact solution.

2. Project $\tilde{p}^{(0)}$ and $\tilde{p}^{l s q}$ onto the characteristic field by multiplying them with the left eigenvectors

$$
\begin{aligned}
& \tilde{\tilde{p}}_{i}^{(0)}=L_{i} \cdot \tilde{p}^{(0)}, \\
& \tilde{\tilde{p}}_{i}^{l s q}=L_{i} \cdot \tilde{p}^{l s q},
\end{aligned}
$$

where $L_{i}$ are the left eigenvectors based on the mean values of the target cell and the normal direction $i$. For triangle, there are three normal directions corresponding to each face of the element. Note: $\tilde{p}^{(0)}:=p^{(0)}$.

3. Evaluate $\tilde{p}_{i}^{(0)^{n e w}}$ for each direction $i$ using $\tilde{\tilde{p}}_{i}^{(0)}$ and $\tilde{\tilde{p}}_{i}^{l s q}$; i.e.,

$$
\tilde{p}_{i}^{(0)^{\text {new }}}=\frac{\omega_{0}}{\gamma_{0}} \tilde{\tilde{p}}_{i}^{(0)}+\left(\omega^{l s q}-\omega_{0} \frac{\gamma^{l s q}}{\gamma_{0}}\right) \tilde{\tilde{p}}_{i}^{l s q} .
$$

4. Project $\tilde{p}_{i}^{(0)^{\text {new }}}$ back into the physical space

$$
p_{i}^{(0)^{n e w}}=R_{i} \cdot \tilde{p}_{i}^{(0)^{\text {new }}}
$$

where $R_{i}$ are the right eigenvectors based on the mean values of the target cell and the normal direction $i$.

5. Obtain the final reconstructed WENO polynomial for the target cell with weighted averaging:

$$
p^{(0)^{\text {new }}}=\frac{\sum_{i} p_{i}^{(0)^{\text {new }}}\left|\Delta_{i}\right|}{\sum_{i}\left|\Delta_{i}\right|},
$$

where $\left|\Delta_{i}\right|$ corresponds to the volume of the neighboring element for face $i$ of the target element. If the face is a boundary face, use the volume of the target element, $\left|\Delta_{0}\right|$. 


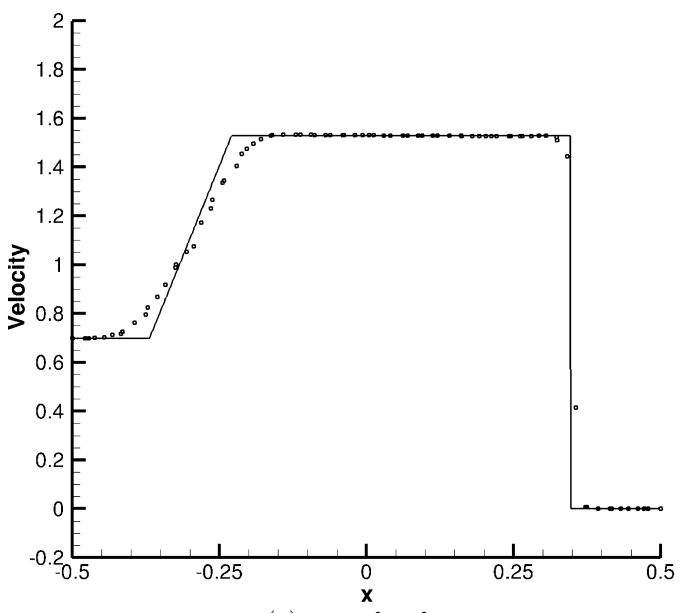

(a) second-order

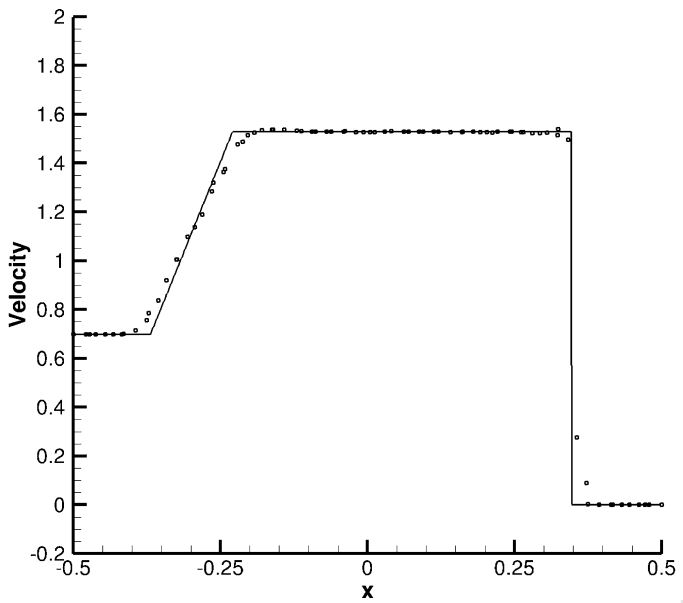

(c) fourth-order

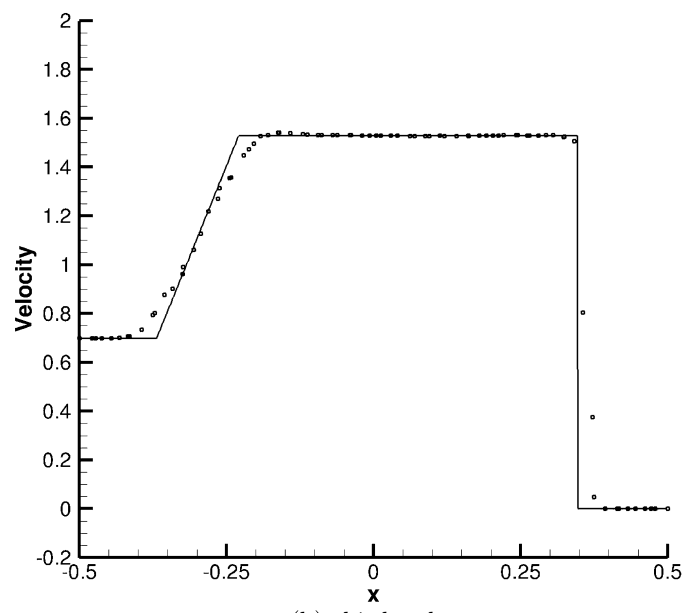

(b) third-order

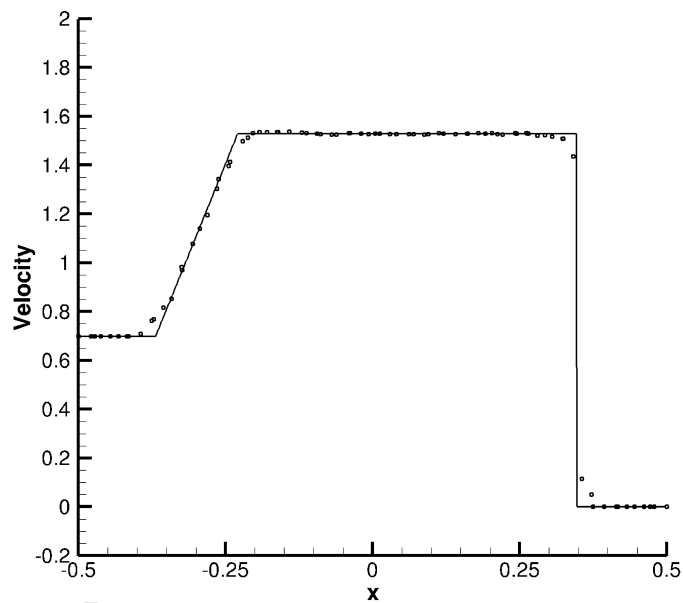

(d) fifth-order

Fig. 12. Predicted velocity profile for the Lax problem with $h / 100$ irregular triangular elements at $t=0.14$, and comparison against the exact solution.

\section{Positivity preserving limiter (PPL) for Euler}

The proposed WENO-DG does not necessary bound the density and pressure within their physical values and thus, a proper bound preserving strategy must be adopted to avoid occurrences of unphysical quantities in time and/or space. The bound preserving limiter must also preserve the formal order of accuracy of the underlying DG scheme. Here, a positivity preserving algorithm of Wang et al. [32] is adopted and applied to the polynomials in conjunction with the WENO polynomial limiters. The presented PPL limiter for Euler equations, Eq. (1), is completely local requiring only information within each element, and therefore, enabling an extremely efficient parallel implementation. Similar to the presented WENO-DG, the given PPL procedure is also nonintrusive and may be applied as a postprocessing step to the polynomials after each time iteration. The step-by-step PPL procedure for DG methods is:

1. Compute the means of the polynomials $\mathbf{w}=(\rho, \rho \mathbf{u}, \rho E)^{T}$ on each element $\Delta$

$$
\bar{w}_{i}=\frac{1}{\Delta} \int_{\Delta} w_{i},
$$

where, $i=0 \ldots(d+1)$ denotes the indices of the vector of conservative variables $\mathbf{w}$, and $d$ refers to the dimension.

2. Construct a new polynomial for density as follows

$$
\hat{\rho}=\theta^{(1)}(\rho-\bar{\rho})+\bar{\rho}, \quad \theta^{(1)}=\min _{x \in q}\left\{1, \frac{\bar{\rho}-\epsilon}{\bar{\rho}-\rho_{q}(x)}\right\},
$$

where $\epsilon$ is a small number, which is taken as $10^{-13}$ in this study, and subscript $q$ denotes values defined on GaussLobatto quadrature points. This step enforces positivity for density. 


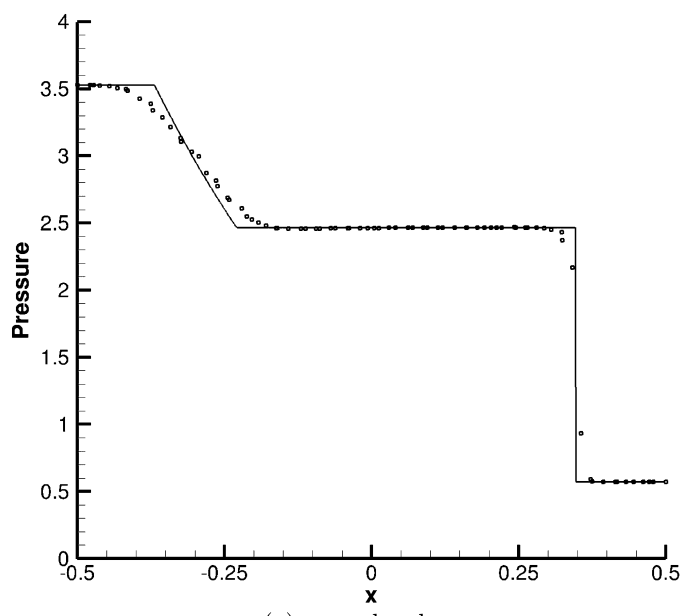

(a) second-order

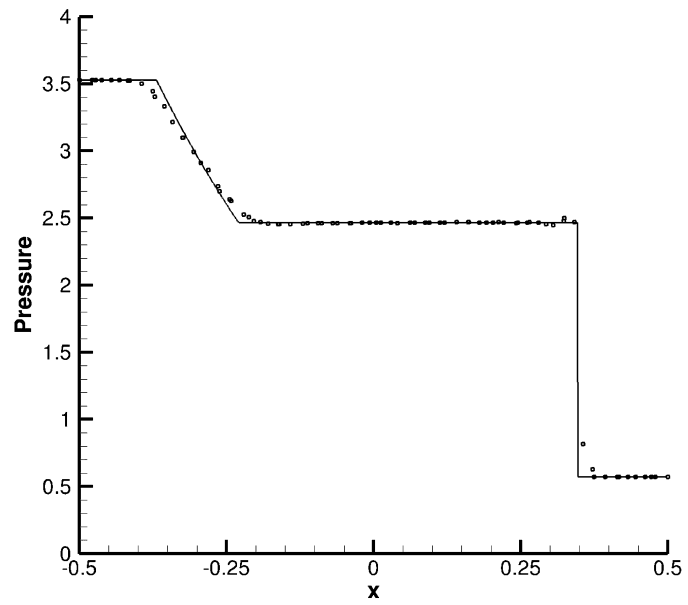

(c) fourth-order

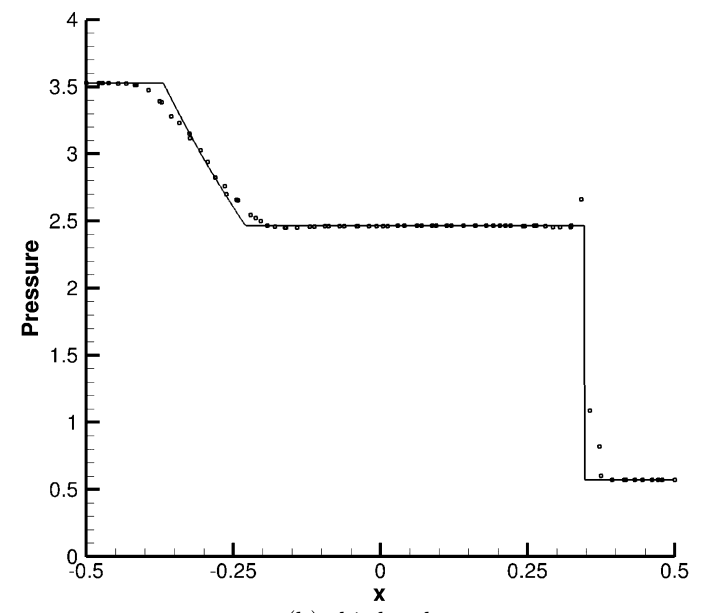

(b) third-order

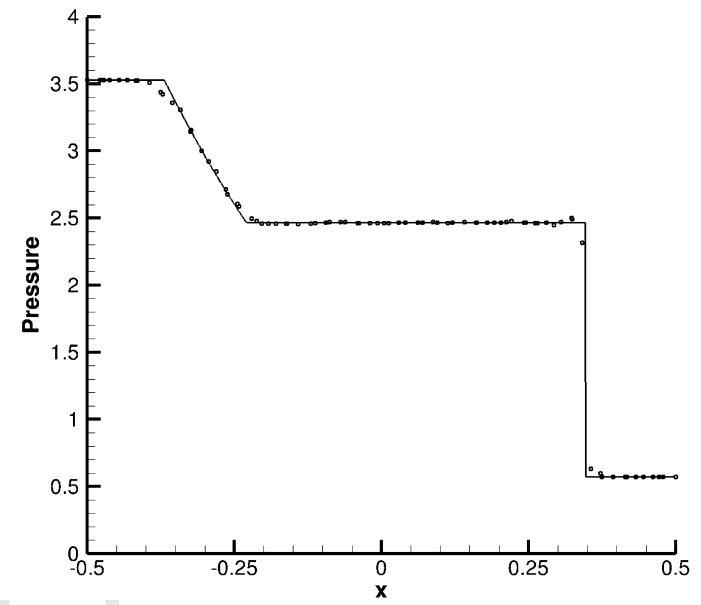

(d) fifth-order

Fig. 13. Predicted pressure profile for the Lax problem with $h / 100$ irregular triangular elements at $t=0.14$, and comparison against the exact solution.

3. Create a new vector of conservative variables $\hat{\mathbf{w}}$ with the new density polynomial $\hat{\rho}$ obtained in the previous step

$$
\hat{\mathbf{w}}=\left(\begin{array}{c}
\hat{\rho} \\
\rho \mathbf{u} \\
\rho E
\end{array}\right) .
$$

4. Compute the following scaling factor for every quadrature point, $q$

$$
\theta_{q}(x)=\left\{\begin{array}{ll}
1 & : p(\hat{\mathbf{w}}) \geq 0 \\
p(\overline{\mathbf{w}}) /\left(p(\overline{\mathbf{w}})-p\left(\hat{\mathbf{w}}_{q}(x)\right)\right) & : \text { otherwise }
\end{array} .\right.
$$

5. Limit the $\hat{\mathbf{w}}$ polynomials to obtain $\tilde{\mathbf{w}}$

$$
\tilde{\mathbf{w}}=\theta^{(2)}(\hat{\mathbf{w}}-\overline{\mathbf{w}})+\overline{\mathbf{w}}, \quad \theta^{(2)}=\min _{x \in q}\left(\theta_{q}(x)\right)
$$

6. Replace $\mathbf{w}$ with the limited polynomials $\tilde{\mathbf{w}}$ after each time iteration. For explicit time schemes such as SSP RK, this procedure is applied after each RK stage.

\section{Numerical results}

The limiters' abilities to achieve the desired order of accuracy is verified first using an inhomogeneous Euler system. A few sample examples are then presented to assess the performance of the proposed limiters. These include the classical Riemann problems (Sod and Lax), the Shu-Osher shock-density interactions, the Mach 3 wind tunnel case of Woodward and Colella [39], a Mach 3 two-strut scramjet, Mach 3 and Mach 10 blunt body problems, and the Mach 10 double Mach 


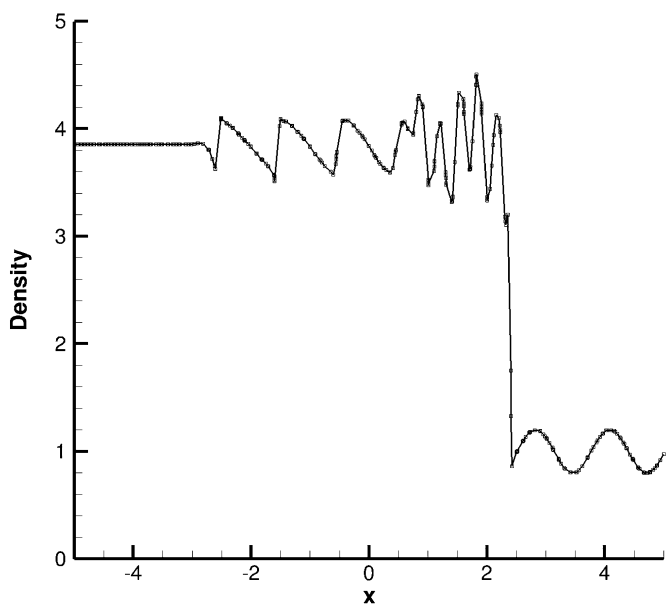

(a) second-order

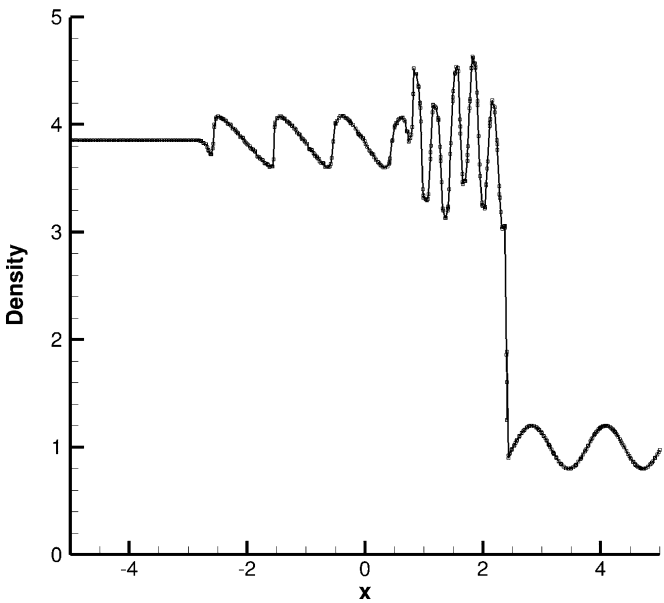

(c) fourth-order

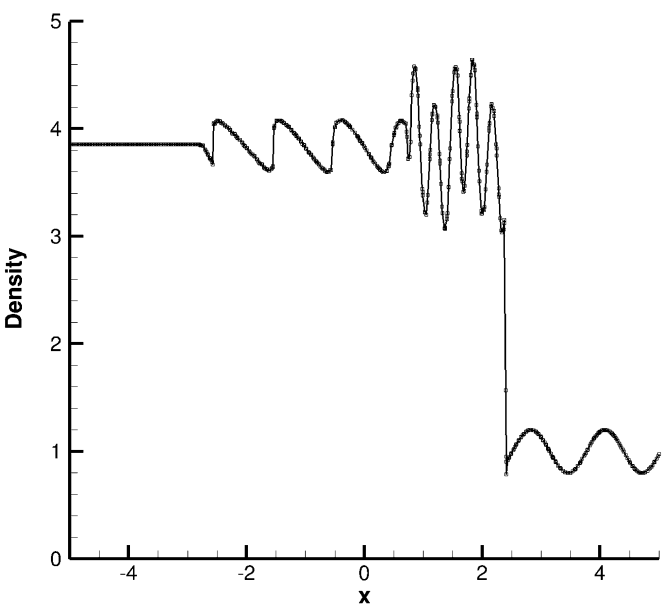

(b) third-order

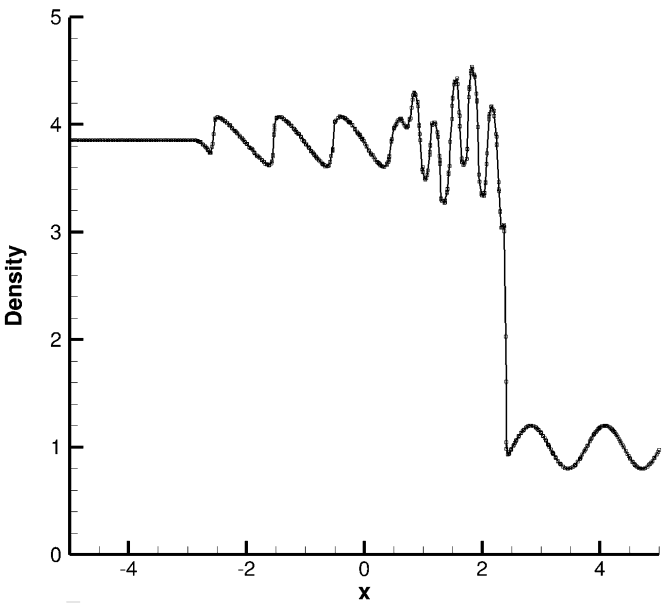

(d) fifth-order

Fig. 14. Predicted density profile for the Shu-Osher problem with $h / 40$ irregular triangular elements at $t=1.8$.

reflection problem. In all the test cases presented here, the corresponding domains are discretized with irregular triangular elements and simulations are carried with a CFL condition that is formally proven to be stable [40] under the following condition

$$
\|\lambda\| \frac{d t}{h} \leq \frac{1}{2(2 k+1)}
$$

where $\|\lambda\|$ is the magnitude of the largest characteristic quantity of the hyperbolic system, $d t$ is the time step, $h$ is the element size, and $k$ is the polynomial order.

For each test case, the proposed WENO-DG schemes are applied and the predicted results are shown for second-, third-, fourth-, and fifth-order solutions. The computed nonlinear weights profiles are also presented.

\subsection{Verification - inhomogeneous Euler}

Consider the two-dimensional Euler equations

$$
\mathbf{w}_{t}+\nabla \cdot \mathbf{f}(\mathbf{w})=\mathbf{S}, \quad \mathbf{w}(\mathbf{x}, t=0)=\mathbf{w}_{0}(\mathbf{x}),
$$

with the vector of conservative variables $\mathbf{w}$ and the source $\mathbf{S}$,

$$
\mathbf{S}=\left(\begin{array}{l}
0.4 \cos (x+y) \\
0.6 \cos (x+y) \\
0.6 \cos (x+y) \\
1.8 \cos (x+y)
\end{array}\right),
$$




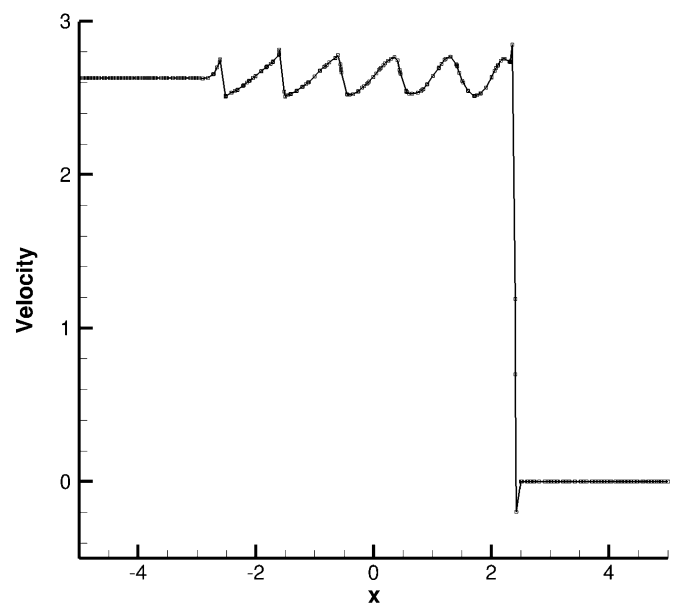

(a) second-order

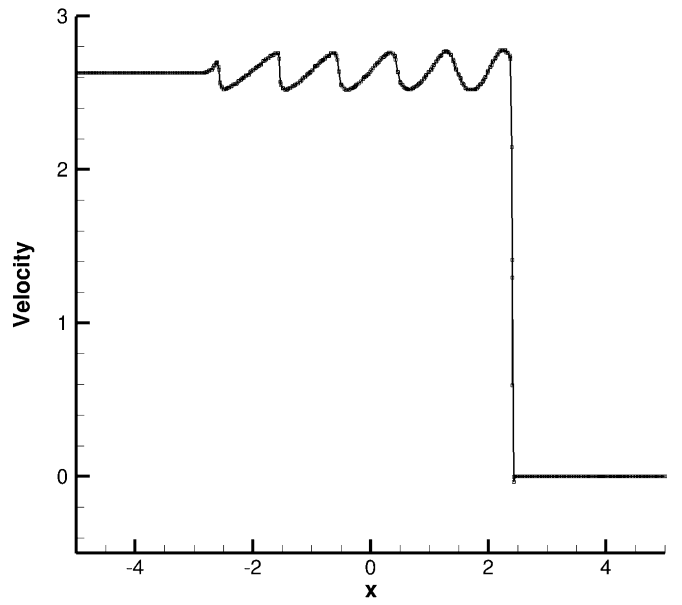

(c) fourth-order

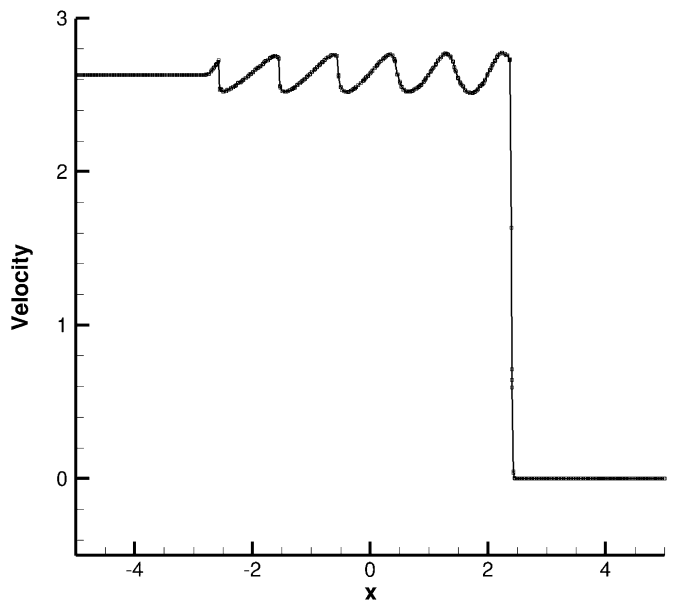

(b) third-order

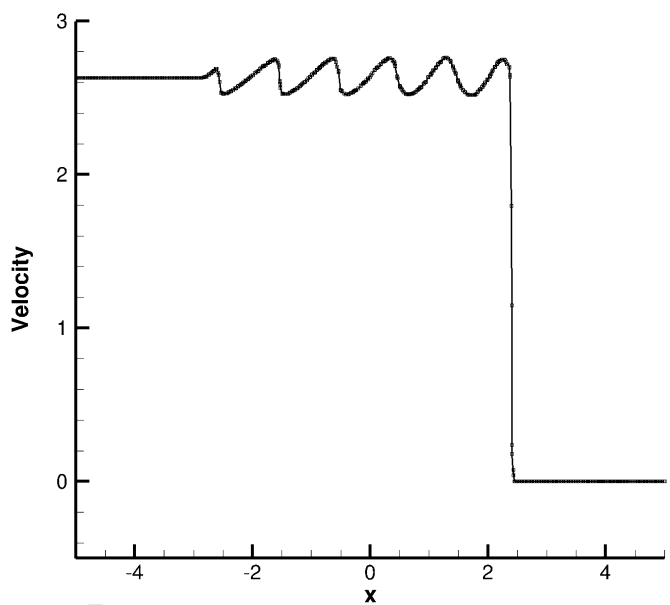

(d) fifth-order

2

$$
\rho=1+0.2 \sin (x+y), \quad u=1, \quad v=1, \quad p=1+0.2 \sin (x+y),
$$

which is imposed on the domain boundaries. A series of randomly generated irregular triangular grids is considered (see Fig. 2), and steady state solutions of the DG $(P k), k=1,2,3,4$, scheme with and without the presented WENO and PPL limiters are obtained. The convergence history plots for third-, fourth-, and fifth-order cases shown in Figs. 3 and 4 indicate that the residuals have settled down to $10^{-12}-10^{-15}$ values (machine zero) for both presented WENO approaches. Fig. 5 shows the difference in residuals history for both explicit SSP $(3,3)$ RK and implicit Euler backward time discretization schemes.

The predicted high-order DG $(P k)$ solutions are also compared with the exact solution on the given grids by computing the $L_{2}$ error. The resulting $L_{2}$ errors are tabulated in Tables 1-4 and shown in Fig. 6 . The $L_{2}$ errors for both WENO limiters are identical to the decimal point shown and therefore only one set of values is given. The WENO and positivity preserving limiters are both applied to all the elements within the domain. The $(k+1)$ th order of accuracy for both DG and the WENO-DG schemes are verified, confirming that the proposed WENO maintains the expected order of accuracy of the underlying DG $(P k)$ scheme. It is also interesting to note that the presented error magnitudes are order of magnitude smaller than the corresponding results reported with the third-, fourth-, and fifth-order WENO-FV schemes in Ref. [31].

\subsection{Riemann problems}

Here, a two-dimensional irregular triangular domain is considered to test the WENO scheme against the classical one-dimensional Sod and Lax problems. The computational domain $(x, y) \in[-0.5,-0.05] \times[0.5,0.05]$ is discretized with triangular elements with a characteristic mesh size of $h / 100$; see Fig. 7. The two states of the gas $(\gamma=1.4)$ are separated 


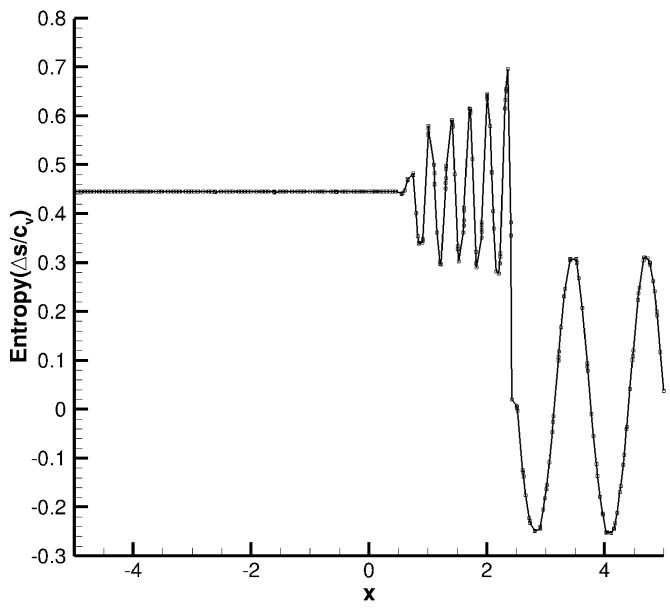

(a) second-order

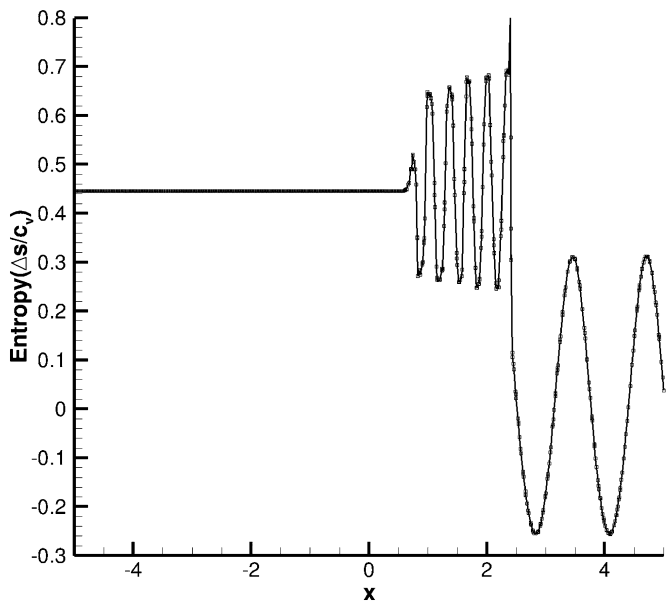

(c) fourth-order

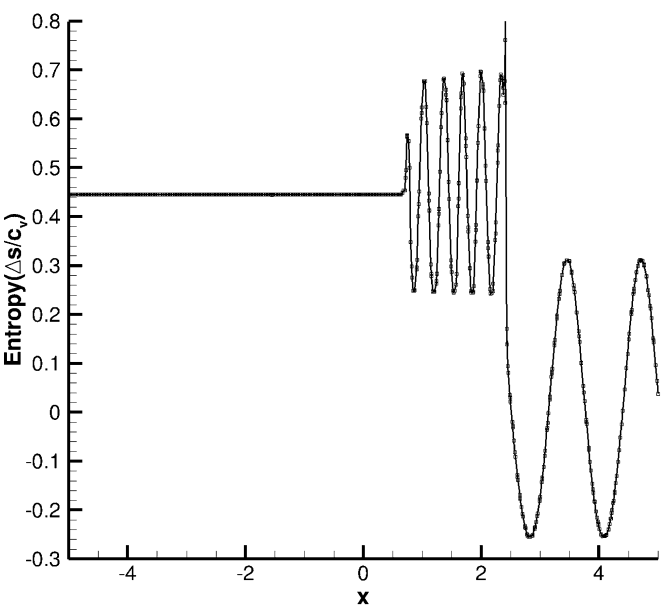

(b) third-order

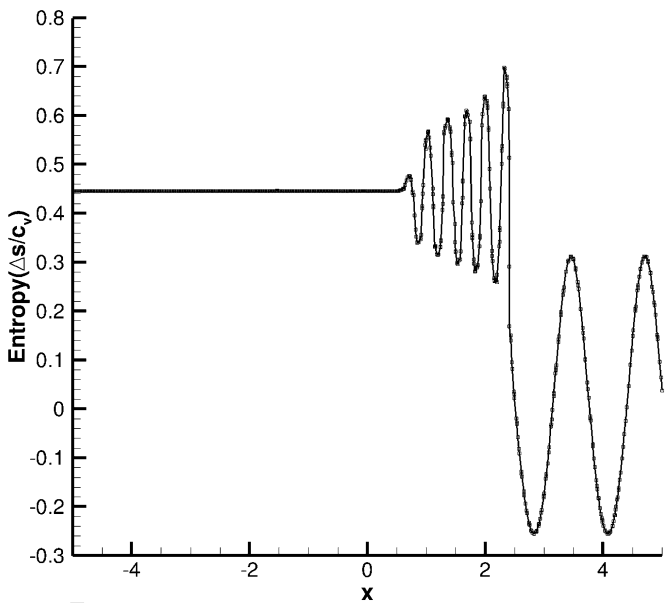

(d) fifth-order

Fig. 16. Predicted entropy profile, $\ln \left(p / \rho^{\gamma}\right)$, for the Shu-Osher problem with $h / 40$ irregular triangular elements at $t=1.8$.

at $x_{d}$ with the initial left and right values given in Table 5. The proposed WENO-DG is then applied to these problems, and the simulations are continued until the $t_{\text {final }}$ is reached.

Figs. 8-13 show the predicted solutions, $(\rho, u, p)$, against the exact Sod and Lax values. The predicted fifth-order WENODG results are comparable with the fifth-order ADER-CWENO scheme of Dumbser et al. [30].

\subsection{Shu-Osher problem}

The one-dimensional idealization of the shock-turbulence interaction suggested by Shu-Osher [41] is considered in a two-dimensional framework. The goal of this test is to assess the proposed limiters in capturing the shock wave and its interactions with the unsteady low frequency density fluctuations and its waves propagations downstream of the shock. The computational domain $(x, y) \in[-5.0,-0.1] \times[5.0,0.1]$ is discretized with triangular elements and a characteristic mesh size of $h / 40$. The domain is initialized as

$$
\left.(\rho, u, v, p)\right|_{t=0}= \begin{cases}(3.857143,2.629369,0 ., 10.33333), & x<4.0 \\ (1 .+0.2 \sin (5 x), 0 ., 0 ., 1 .), & x \geq 4.0 .\end{cases}
$$

This corresponds to a Mach 3 shock $(\gamma=1.4)$ interacting with the sine waves density field. The results at $t=1.8$ are presented in Figs. 14-16. The fine structured of the shock-density wave interactions are clearly captured by the proposed limiter. 


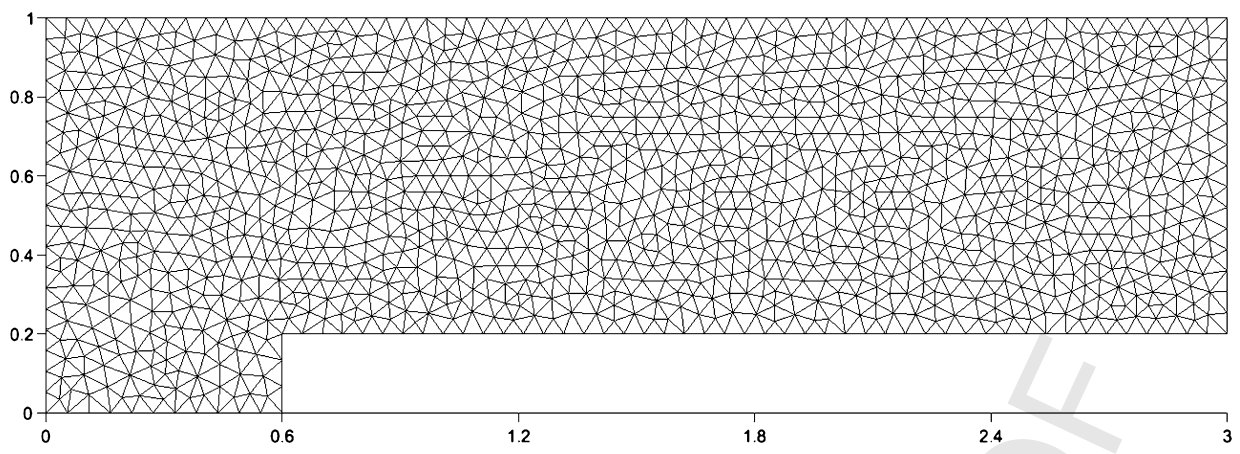

Fig. 17. The geometry of the Mach 3 wind tunnel forward facing step with a sample irregular grid.

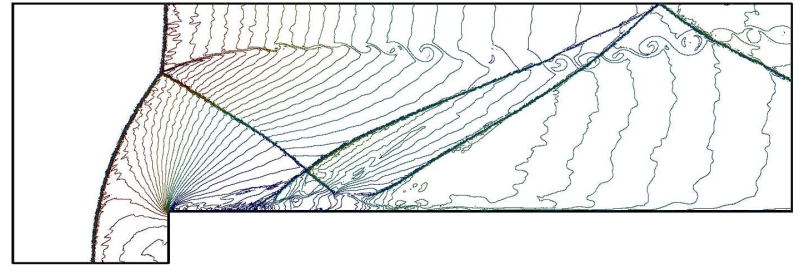

(a) second-order

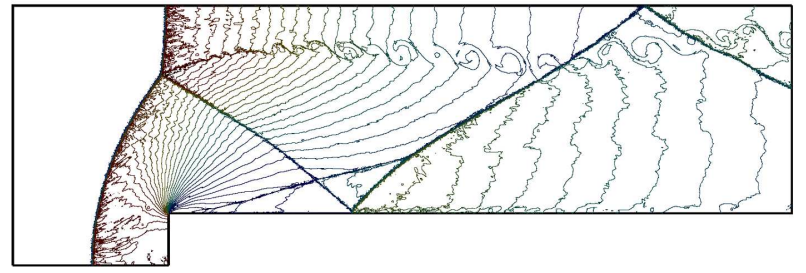

(c) fourth-order

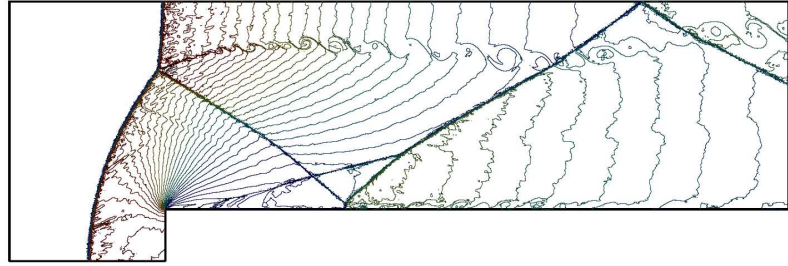

(b) third-order

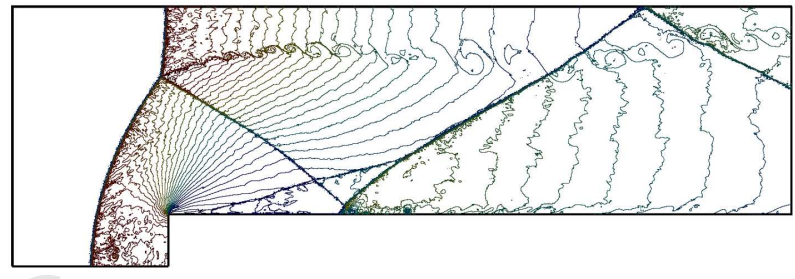

(d) fifth-order

Fig. 18. High-order DG with proposed WENO and positivity preserving with irregular triangular elements $(h=1 / 160)$ for the forward facing step problem. Shown are 30 equidistance density contour lines at $t=4.0$ from 0.32 to 6.15 . The corner step singularity neither in the scheme nor by the grid is treated to remove the known artifacts that is present in the second-order result. These artifacts are largely removed in the higher-order results.

\subsection{Mach 3 forward facing step}

Consider the forward facing step problem that was originally proposed by Woodward and Colella [39]. This test is often referred to as the Mach 3 wind tunnel test. The computational domain is shown in Fig. 17. The initial conditions correspond to a uniform flow moving to the right with Mach $3,(\rho, u, v, p)=(1.4,3.0,0.0,1.0)$. The inflow condition is imposed to the left boundary while a do-nothing boundary condition is set to the right boundary. A reflecting boundary condition is applied to other surfaces, and the flow is initialized with the left boundary values. The domain (see Fig. 17) is discretized using irregular triangular elements with characteristic element size of $h / 160$. The high-order solutions at $t=4.0$ are presented in Fig. 18. Note that no modification, neither to the scheme nor to the grid resolution, is applied to the corner singularity. The corner step singularity is known to produce an erroneous entropy layer in addition to spurious Mach stem at the bottom wall. These artifacts are clearly present in the presented second-order result; the predicted higher order solutions are almost free from these artifacts.

\subsection{Scramjet}

Consider a two-strut scramjet (see Fig. 19 and Table 6) with a Mach 3 inflow imposed on the left surface boundary. For this test, the second proposed approach produced nearly machine zero residuals as illustrated in Fig. 20. The profiles of the computed nonlinear weights along the centerline of the two-strut scramjet are shown in Fig. 21 . The corresponding density contours in the range of 1.5 and 8.0 are shown in Fig. 22. These results are in agreement with the previously published results [42-44] on a similar configuration; the exact geometrical information used in generating the previously published articles was not known to the authors. 


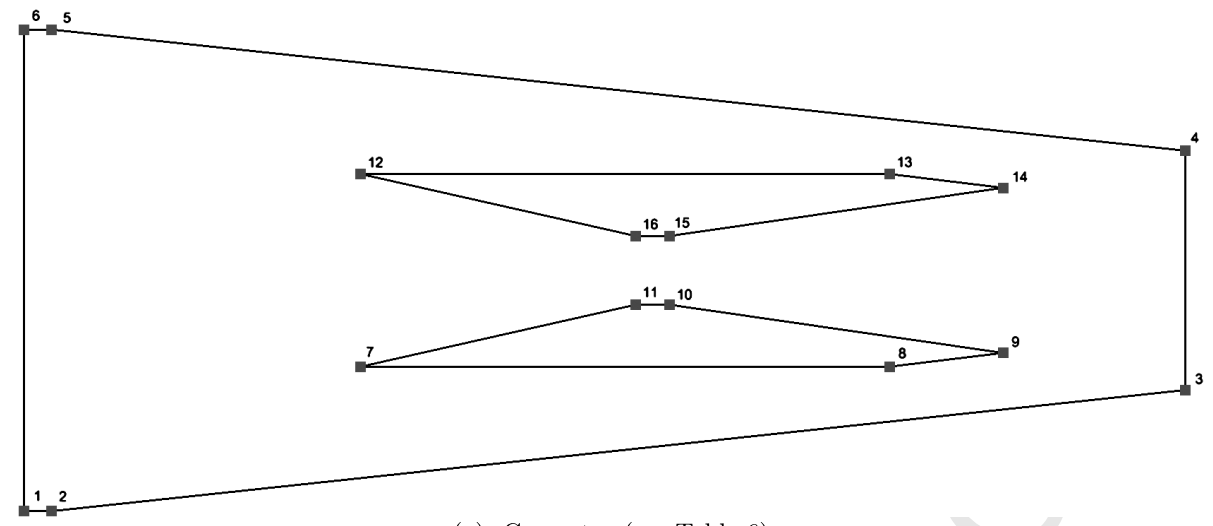

(a) Geometry (see Table 6)

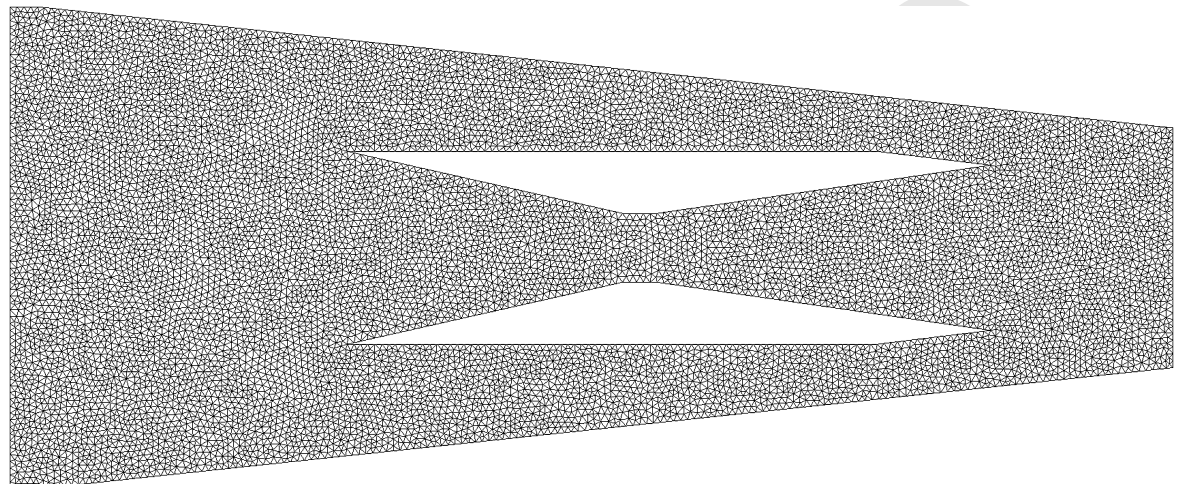

(b) Mesh

Fig. 19. The geometry of the two-strut scramjet test case with a sample irregular grid with 10,000 vertices. Coordinates of the lower half portion of the geometry is given in Table 6 .

Table 6

The coordinates of the lower half portion of the two-strut scramjet geometry shown in Fig. 19.

\begin{tabular}{lllllllll}
\hline Points & 1 & 2 & 3 & 7 & 8 & 9 & 10 & 11 \\
\hline$(x, y)$ & $(0,-3.5)$ & $(0.4,-3.5)$ & $(16.9,-1.74)$ & $(4.9,-1.4)$ & $(12.6,-1.4)$ & $(14.25,-1.2)$ & $(9.4,-0.5)$ & $(8.9,-0.5)$ \\
\hline
\end{tabular}

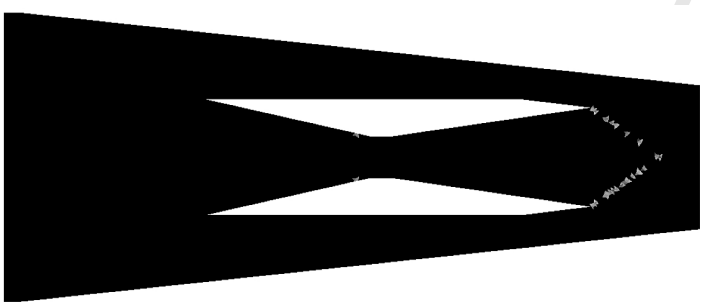

(a) second-order

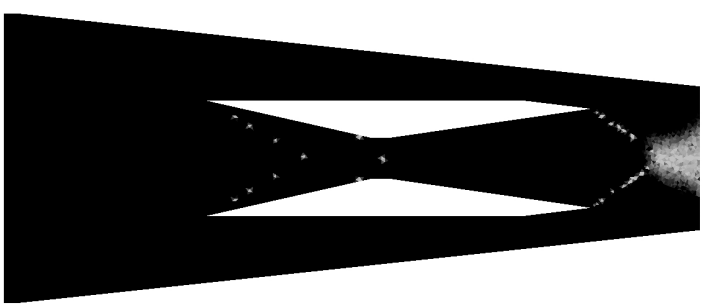

(c) fourth-order

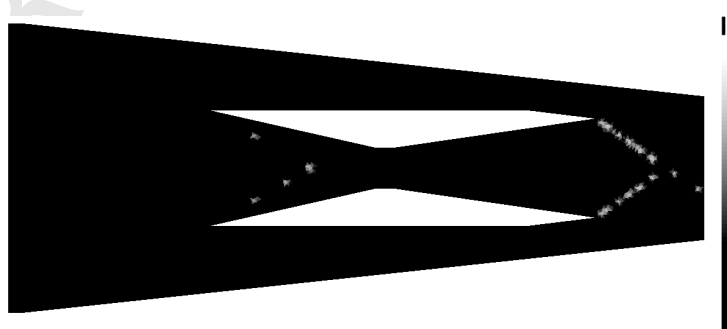

(b) third-order

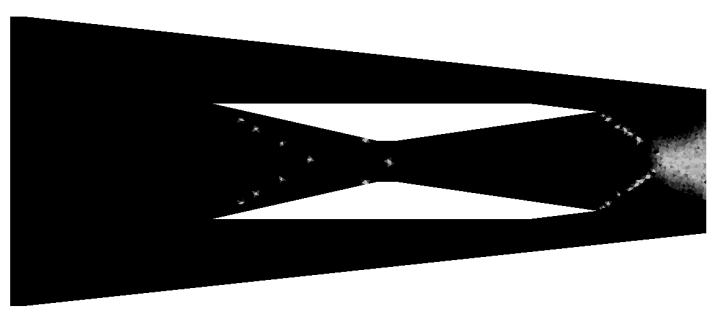

(d) fifth-order
|Residual |

产 $1.00 \mathrm{e}-03$

$1.00 \mathrm{e}-04$

$1.00 \mathrm{e}-05$

$1.00 \mathrm{e}-06$

$1.00 \mathrm{e}-07$

$1.00 \mathrm{e}-08$

$1.00 e-09$

$1.00 e-10$

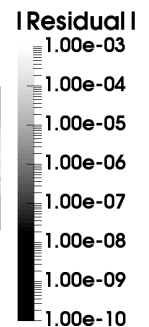

$1.00 e-10$

Fig. 20. Absolute value of the elemental residuals obtained with the second proposed WENO approach for the Mach 3 two-strut scramjet test case.
8

10

11

12

13

14

15

16

17

18

19

20

21

22

23

24

25

26

27

28

29

30

31

33

34

35

36

37

38

39

40

41

42

43

44

45

46

47

48

49

50

51

52

53

54

55

56

57

58

59

60

61 


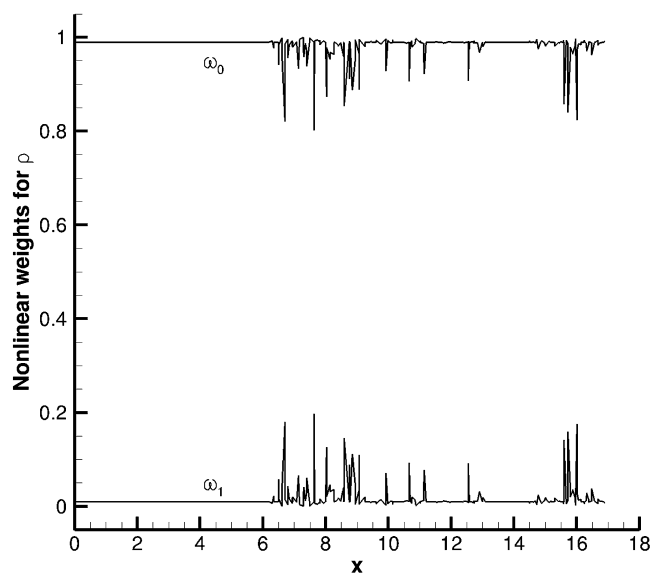

(a) Density

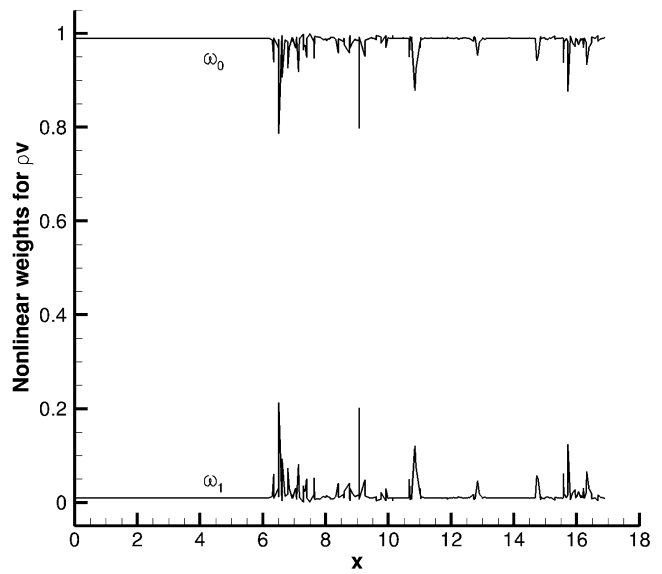

(c) y-momentum

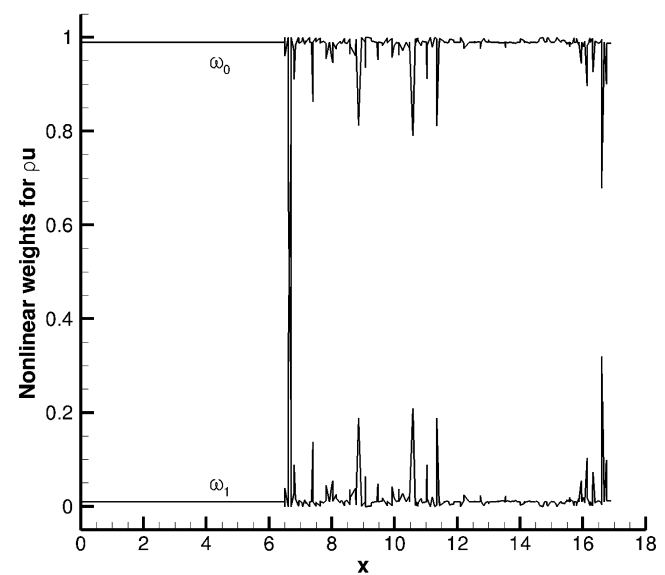

(b) x-momentum

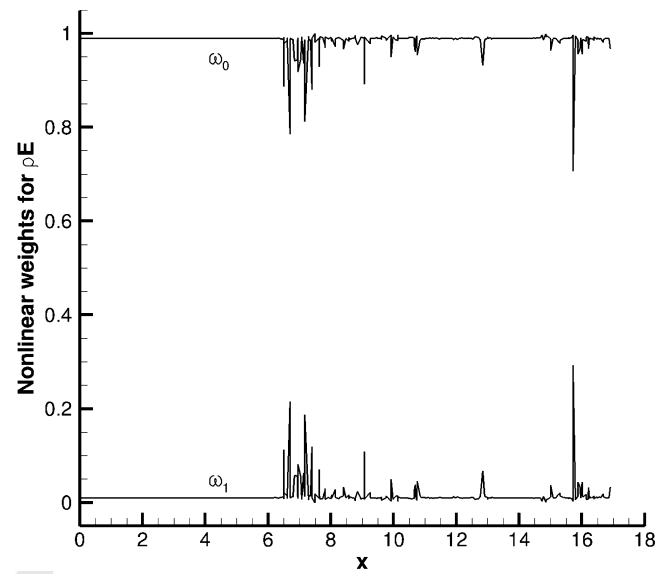

(d) Energy

Fig. 21. Nonlinear weights along the centerline of the Mach 3 two-strut scramjet problem.

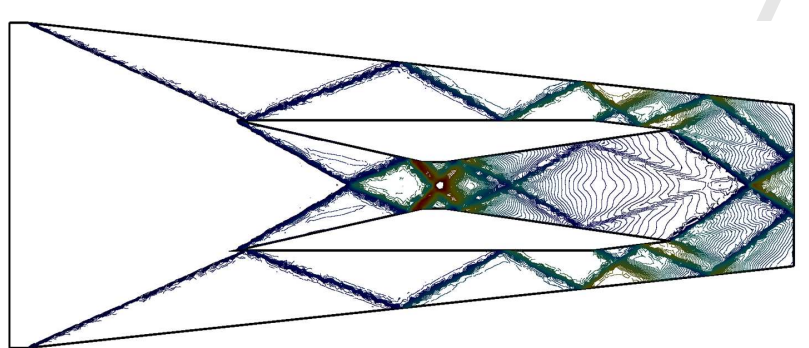

(a) second-order

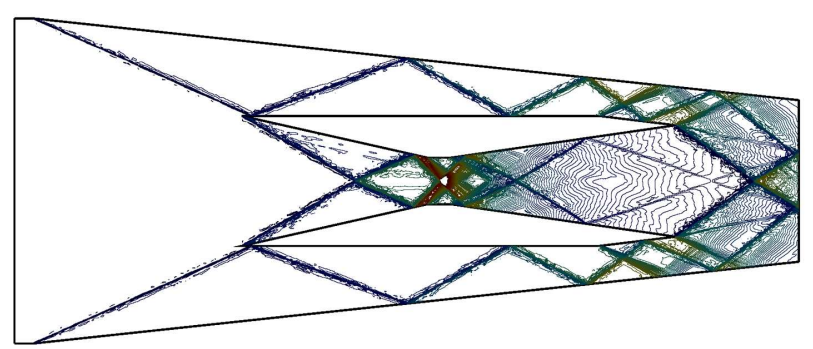

(c) fourth-order

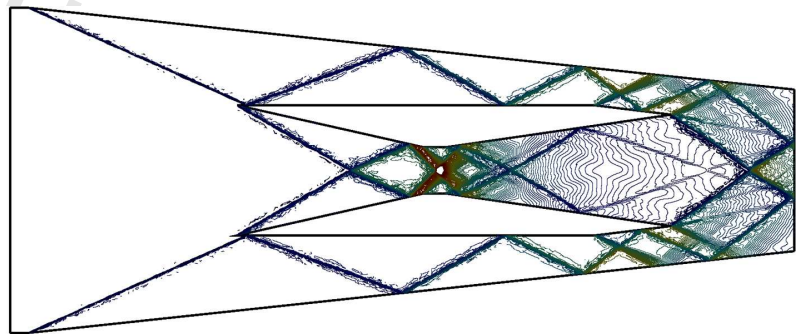

(b) third-order

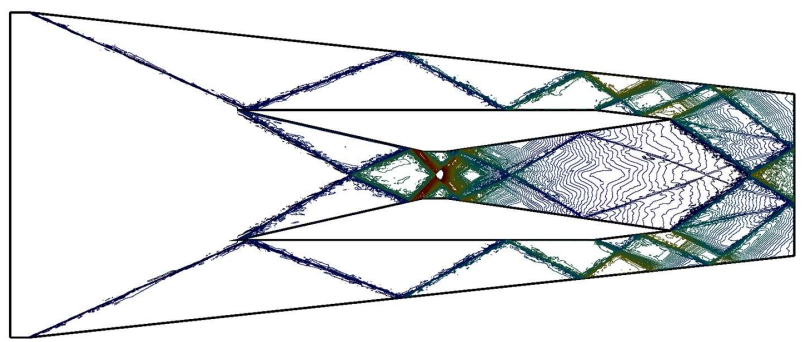

(d) fifth-order

Fig. 22. Hundred equally spaced density contours in the range of 1.5 and 8.0 using the second presented WENO approach for the Mach 3 two-strut scramjet test case with 10,000 unadapted irregular triangular elements. 

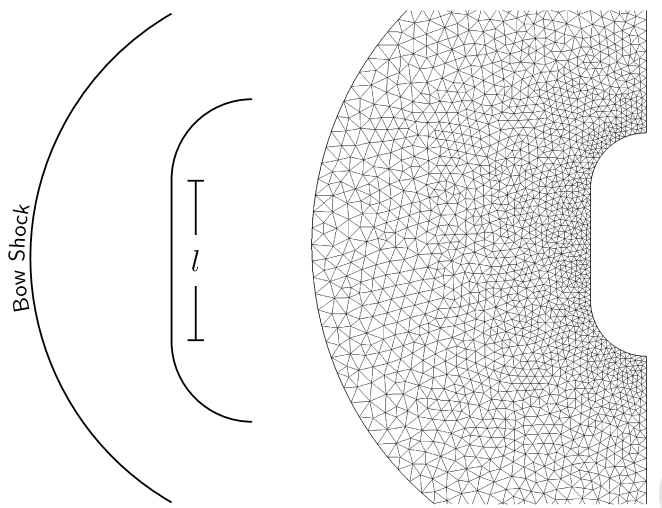

Fig. 23. Schematic of the blunt body geometry and a sample irregular grid.

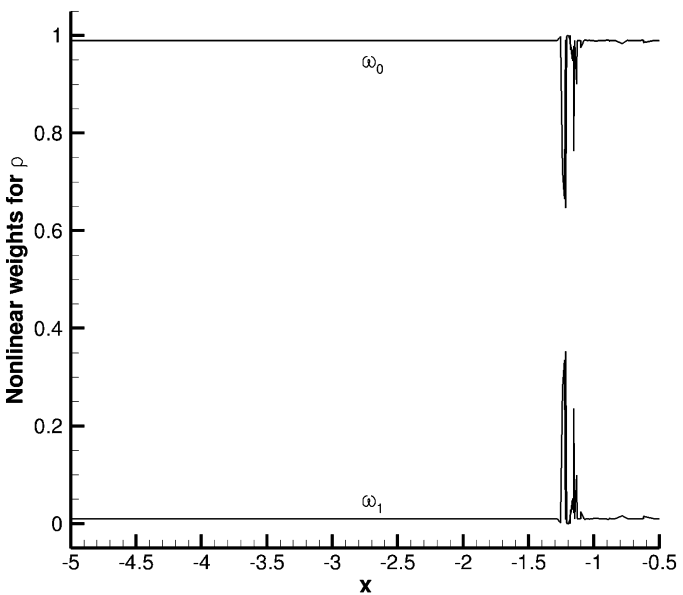

(a) Density

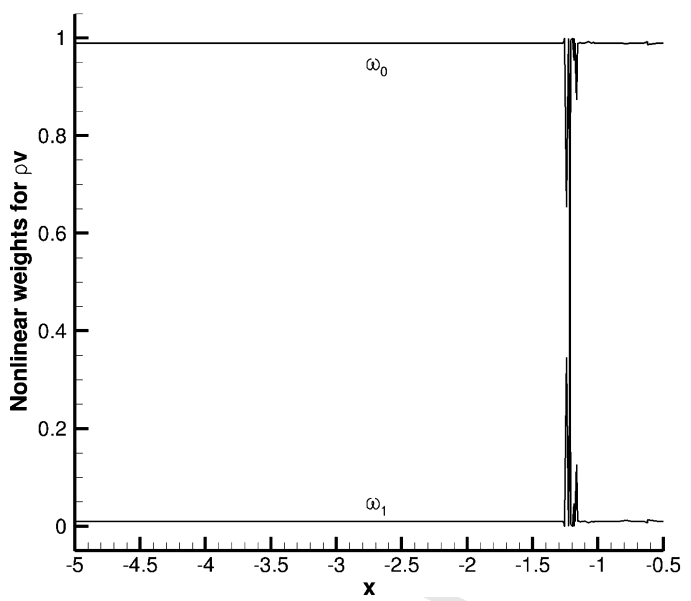

(c) y-Momentum

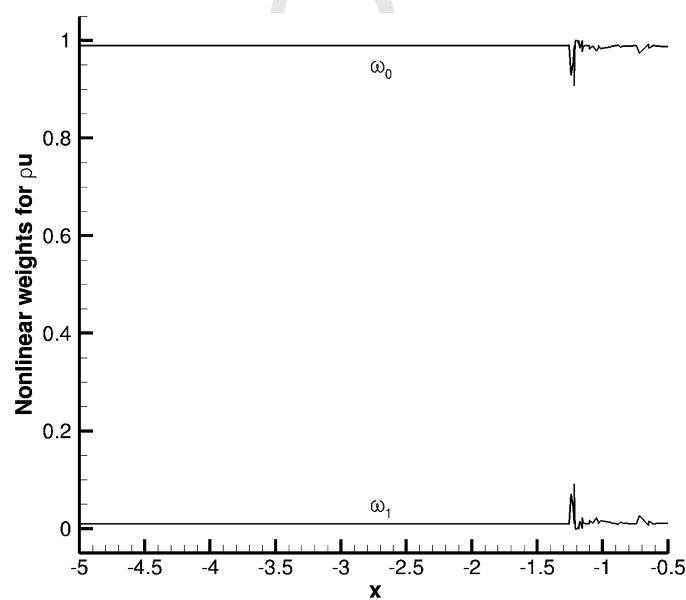

(b) x-Momentum

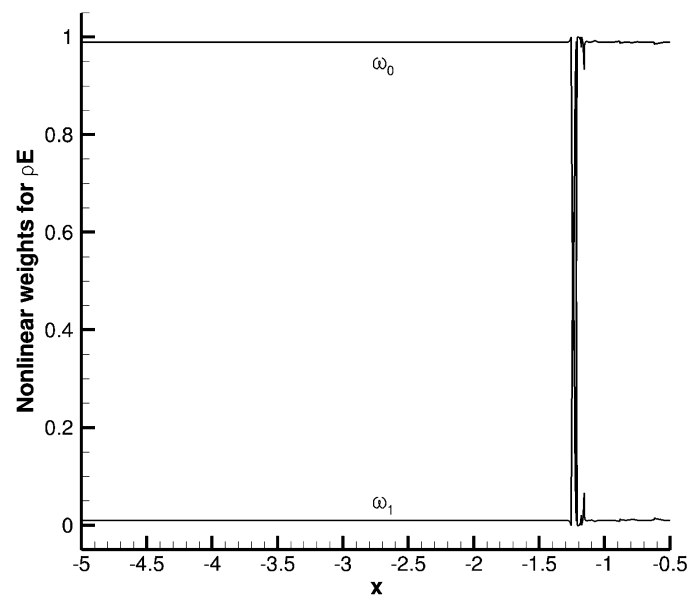

(d) Energy

Fig. 24. Nonlinear weights along the stagnation line of the Mach 10 blunt body problem.

\subsection{Mach 3 and 10 Blunt body flows}

Consider a bluff body in an inviscid compressible flow traveling at Mach 3 and Mach 10. The free stream condition is set to $(\rho, u, v)=(1.4,3,0)$ with $\gamma=1.4$, and a reflecting boundary condition is imposed on the solid surface. The bluff body (see Fig. 23) consists of a flat face with a unit length $l$, and two curved shoulders with $l / 2$ radii.

The plots of the nonlinear weights along the stagnation line for each of the governing equations are shown in Fig. 24 . Density contour lines are shown in Fig. 25 for both Mach 3 and Mach 10 flows. 


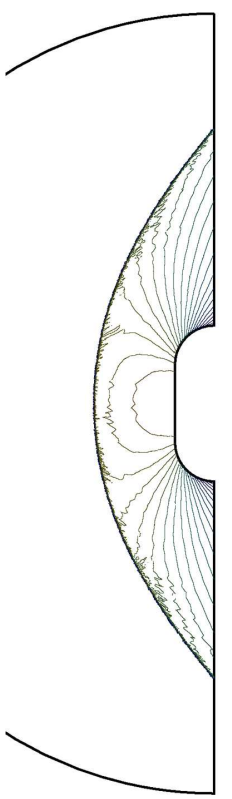

(a) second-order

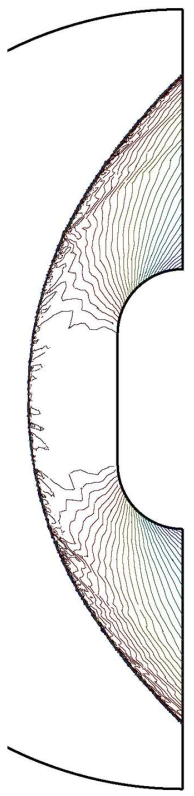

(e) second-order

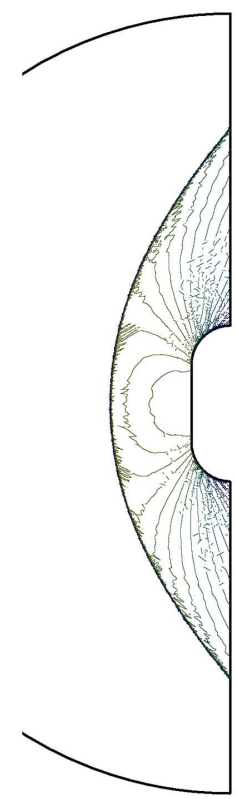

(b) third-order

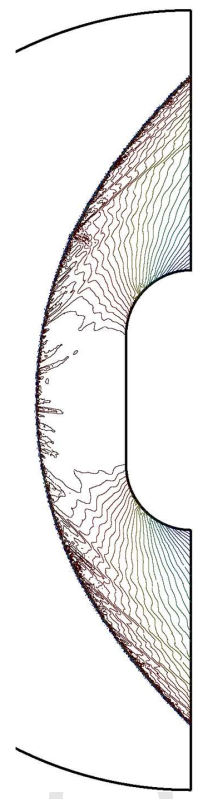

(f) third-order

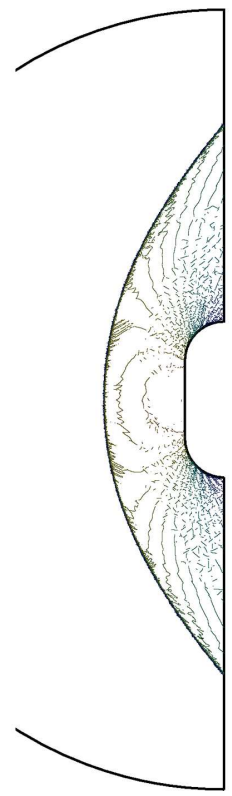

(c) fourth-order

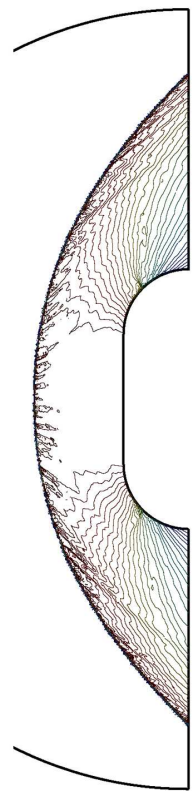

(g) fourth-order

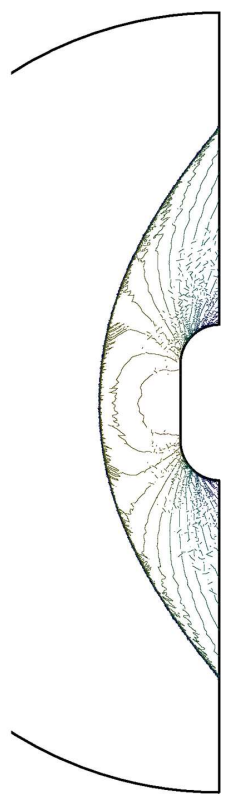

(d) fifth-order

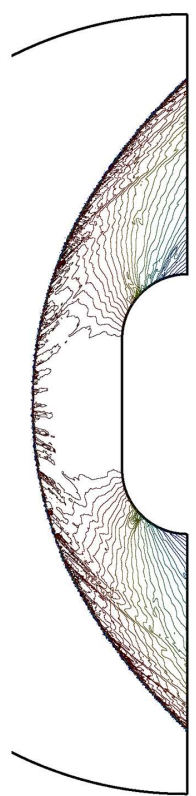

(h) fifth-order

Fig. 25. Thirty equally spaced density contours in the range of 1 and 8 for the Mach 3 (top row) and Mach 10 (bottom row) blunt body test cases.

The strong shocks are correctly captured but there exists some waviness in the density contour lines, particularly when the polynomial order and the intensity of the shock are increased. However, these striation-like patterns, which are due to irregularity of the grid elements in the vicinity of the shock, appear to have minimal effects on the surface quantities as illustrated in Fig. 26. Further improvement to the proposed WENO is needed to reduce these striation-like patterns in the post shock regions. This will be reported in future studies.

\subsection{Double Mach reflection test case}

The double Mach reflection problem is originally proposed by Woodward and Colella [39] as a benchmark test for Euler solvers. The problem consists of a Mach 10 shock front that meets a 30-deg inclined ramp. The shock front initially sits at $x_{0}=1 / 6$, and makes a 60-deg angle with the reflecting wall (x-axis). The properties of the undisturbed air $(\gamma=1.4)$ ahead of the shock are $\rho=1.4$ and $p=1$. Hence, the shock speed is $\left|u_{s}\right|=10$. As it is a customary, this problem is solved 


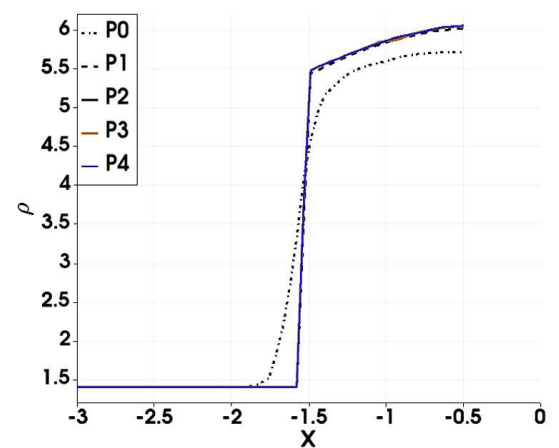

(a) Density

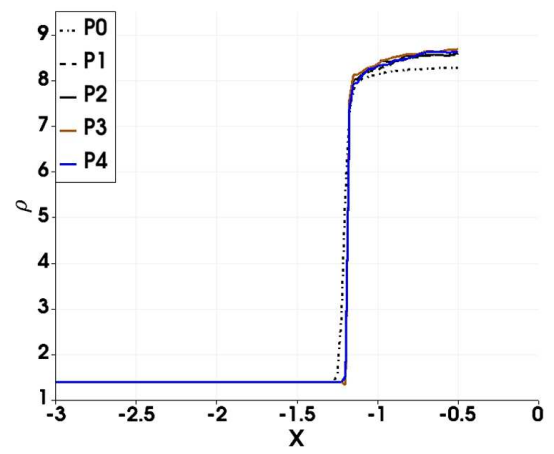

(d) Density

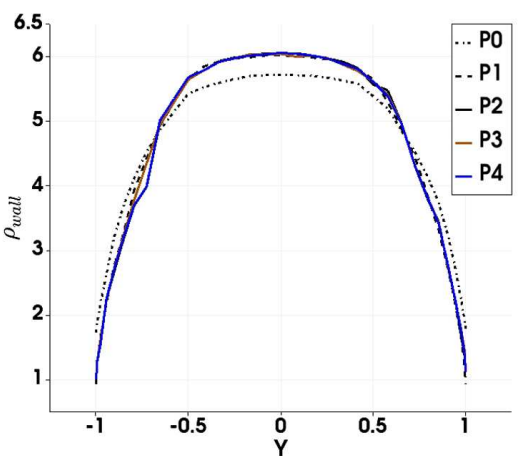

(b) Surface density

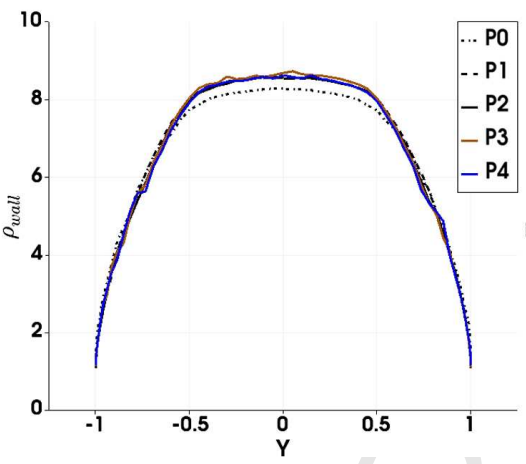

(e) Surface density

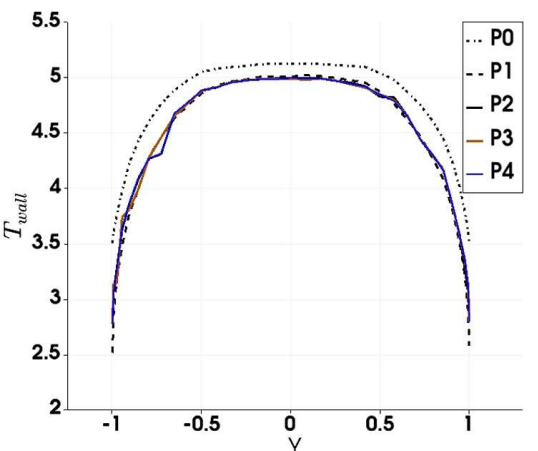

(c) Surface temperature

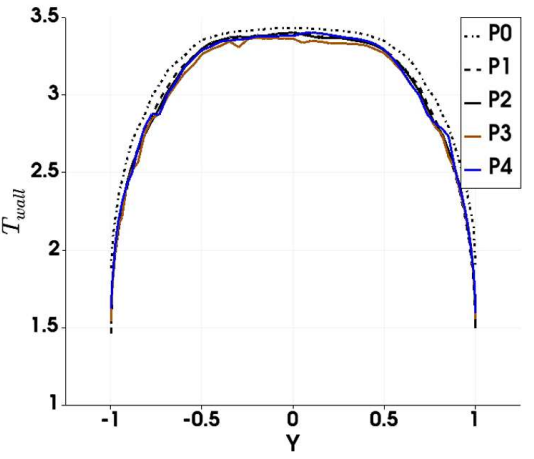

(f) Surface temperature

Fig. 26. Top row: Mach 3. Bottom row: Mach 10; a,d) density profile along a line normal to the stagnation point, b,e) surface density, and c,f) surface temperature. Data are extracted from a low-order visualization.

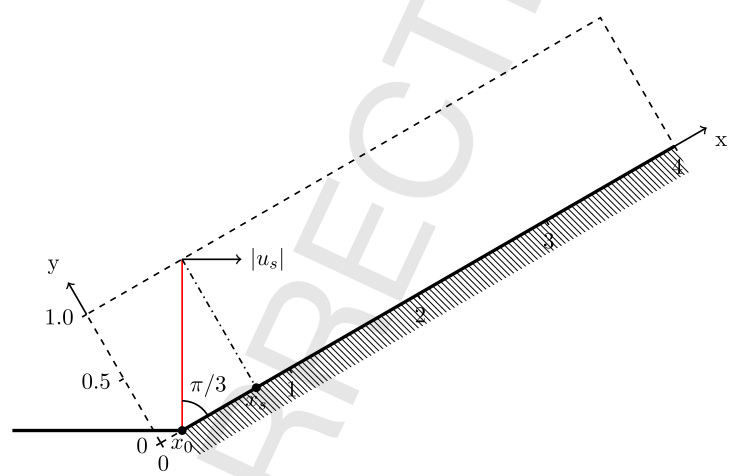

Fig. 27. Schematic of the double Mach reflection, and the computational domain $(x, y) \in[0,4] \times[0,1]$ with the ramp-aligned coordinate system.

in a computational domain $(x, y) \in[0,4] \times[0,1]$ with a coordinate system that is aligned with the ramp; see Fig. 27. The domain is discretized with irregular triangular elements and a mesh size of $h=1 / 200$.

Considering the ramp-aligned coordinate system, the problem is therefore setup with the following initial condition:

$$
\left.(\rho, u, v, p)\right|_{t=0}= \begin{cases}(8.0,+8.25 \cos (\pi / 6),-8.25 \sin (\pi / 6), 116.5), & x<x_{s}, \\ (1.4,0.0,0.0,1.0), & x \geq x_{s},\end{cases}
$$

where the shock position is computed as,

$$
x_{S}(y)=x_{0}+y \tan (\pi / 6) \text {. }
$$

For the boundary conditions, the post-shock values are imposed for the short region from $x=0$ to $x_{S}$ along the lower boundary at $y=0$, while for the rest of the lower boundary, a reflecting boundary condition is imposed. This ensures that the reflecting shock is attached to the wall. The initial post-shock condition is also assigned at the left boundary along the $x=0$ axis. The boundary condition on the upper boundary along the $y=1$ axis consists of both pre- and post-shock values. This boundary condition is imposed based on the exact positioning of the traveling Mach 10 shock; i.e., $x_{s} \mid y=1+$ 


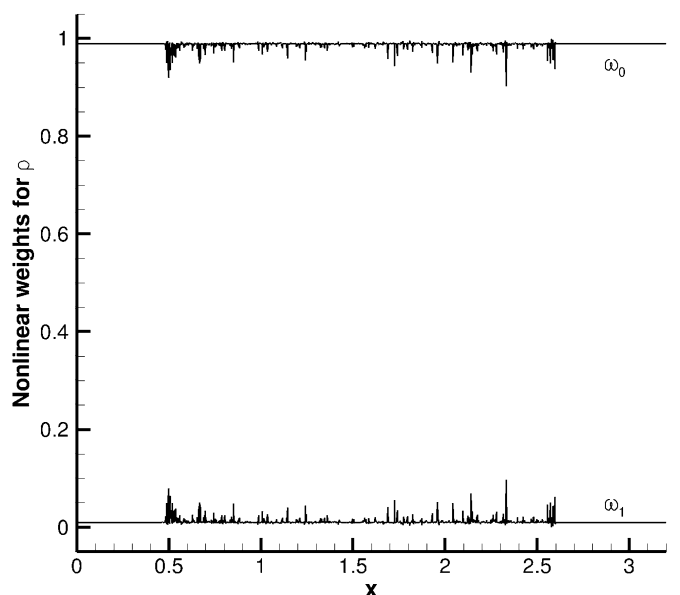

(a) Density

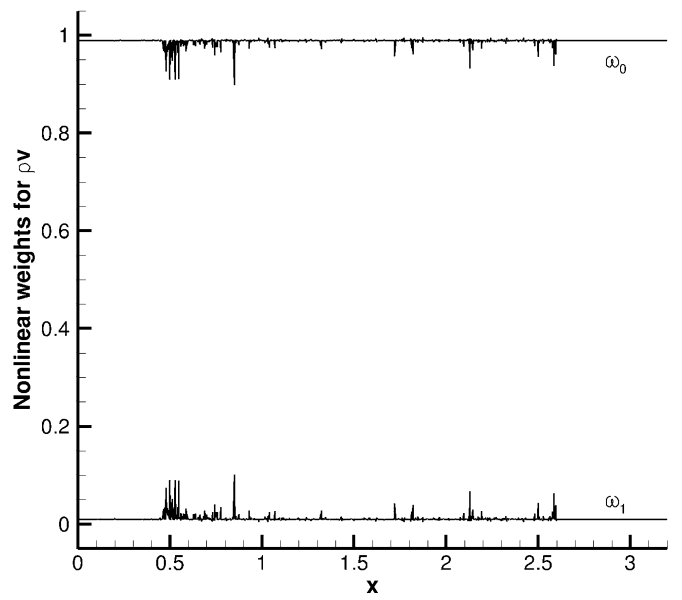

(c) y-momentum

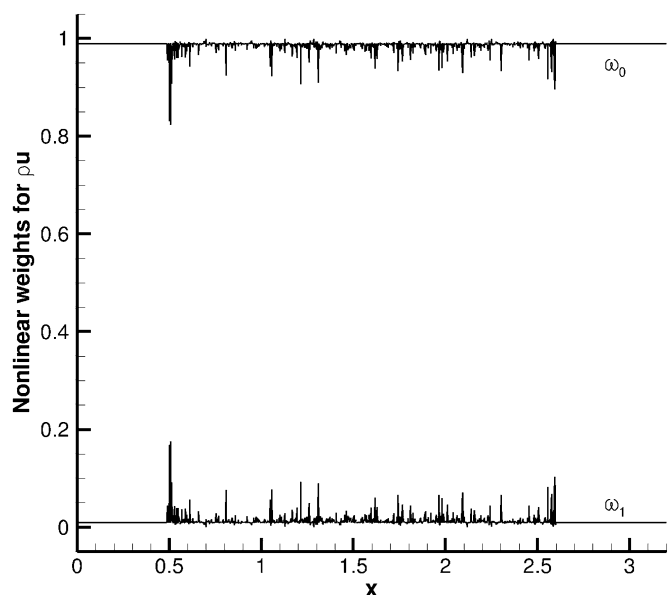

(b) x-momentum

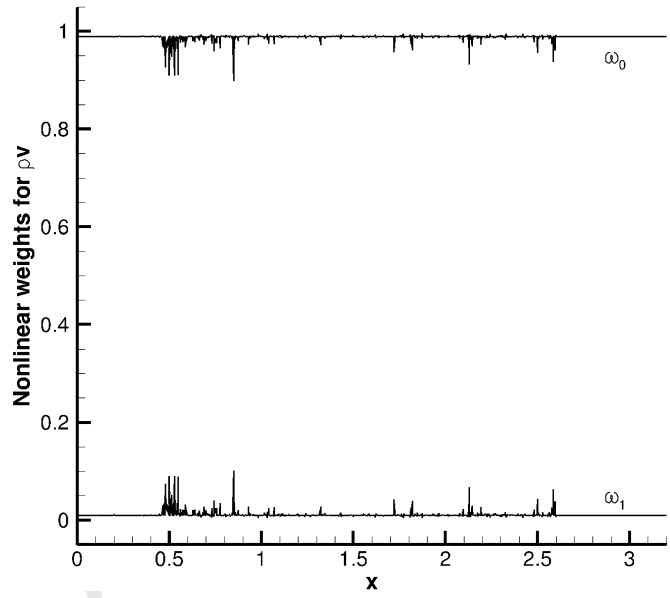

(d) Energy
1

$\left|u_{s}\right| / \cos (\pi / 6) t$. The readers are referred to Ref. [45] for discussions on the effects of the upper boundary condition and the computational domain size on numerical artifacts in the predicted solutions; such effects are not investigated in this study.

The computed nonlinear weights along the $y=0.3$ is plotted in Fig. 28. The density contours for a truncated domain $(x, y)=[0,3.2] \times[0,1]$ (the computational domain extends to $x=4$ ) with 41 equally spaced isolines from 1.5 to 22.5 at $t=0.2$ are shown in Fig. 29 for the second-, third-, fourth-, and fifth-order WENO-DG. The closeup views of the triple Mach points region, $(x, y) \in[2,2.9] \times[0,0.6]$, are shown in Fig. 30. Clearly, the complexity of the triple Mach points is captured by the proposed limiters on the irregular triangular mesh. The quality of the solution improves with increasing the polynomial order on the same identical irregular triangular mesh. The resolution of the curled flow structures along the primary slip line, which is caused by its interactions with the secondary reflected shock emanating from the secondary triple point, is often used for judging the quality of the numerical scheme (see Ref. [45] for definitions of the primary and the secondary triple points and slip lines). The presented results provide a remarkable curled flow structures compared to the solutions reported by Hu and Shu [21], Zhu et al. [46], and Dumbser et al. [30]. Further improvement in the WENO could reduce the noise in the predicted contour lines. Grid adaptation could also enhance the results further.

\section{Concluding remarks}

Two compact WENO limiters were proposed for DG schemes for irregular triangular elements, and detailed step-by-step construction procedures were outlined. The WENO-DG limiters were bounded with a compact bound-preserving limiter for the Euler equations. It was verified that the proposed bounded WENO-DG polynomial limiters preserve the expected order of accuracy of the underlying DG schemes when they are applied to smooth regions. A few test cases, including the classical Riemann problems, Shu-Osher shock-turbulence interaction, scramjet, blunt body, and double Mach reflection problems, involving strong shocks were performed, and the results for second-, third-, fourth-, and fifth-order compact and bounded WENO-DG are presented. It was shown that the second proposed WENO-DG is more effective for some problems in 


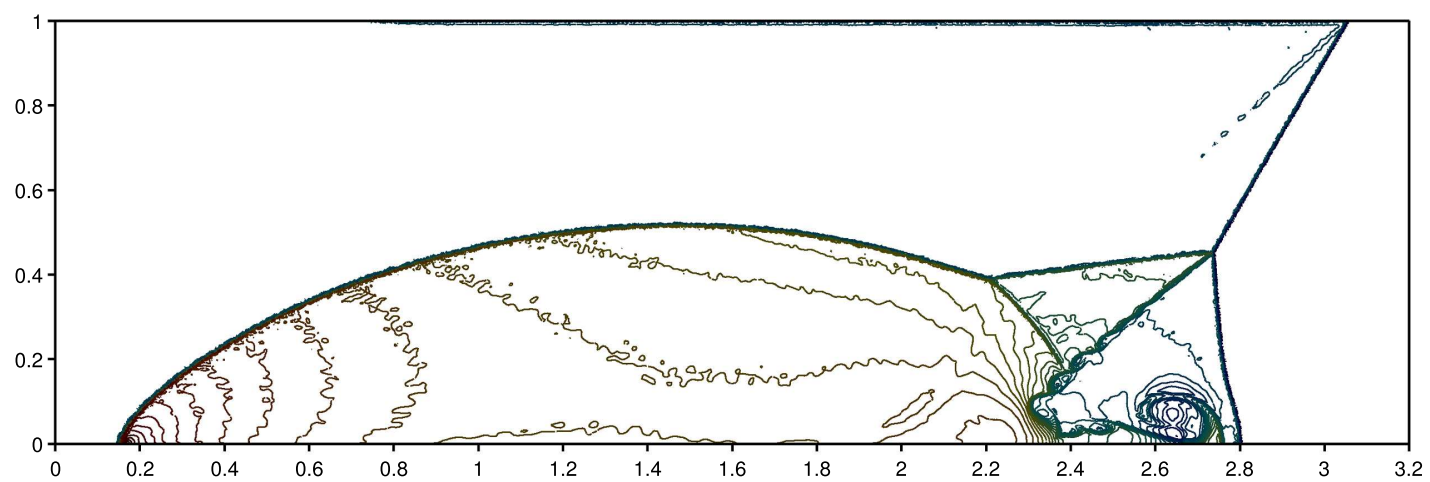

(a) second-order

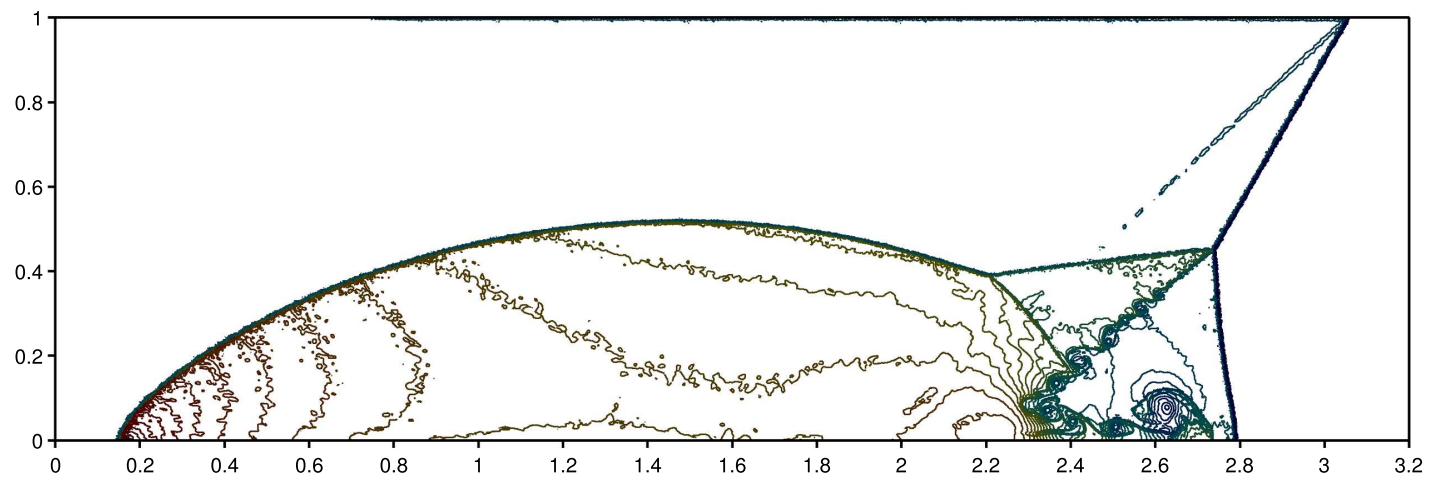

(b) third-order

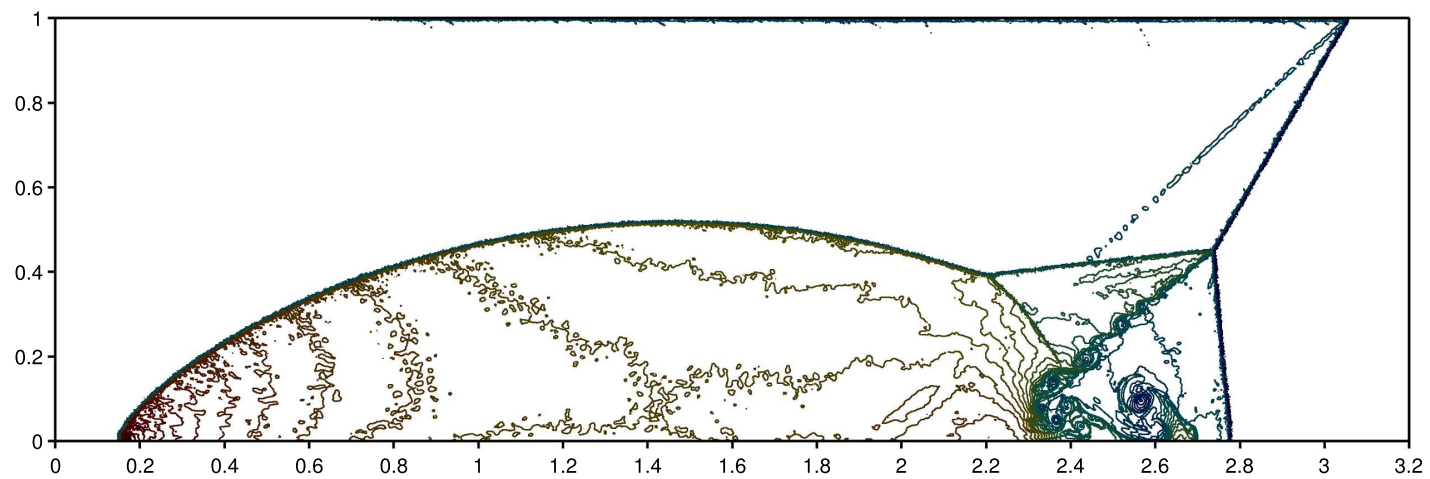

(c) fourth-order

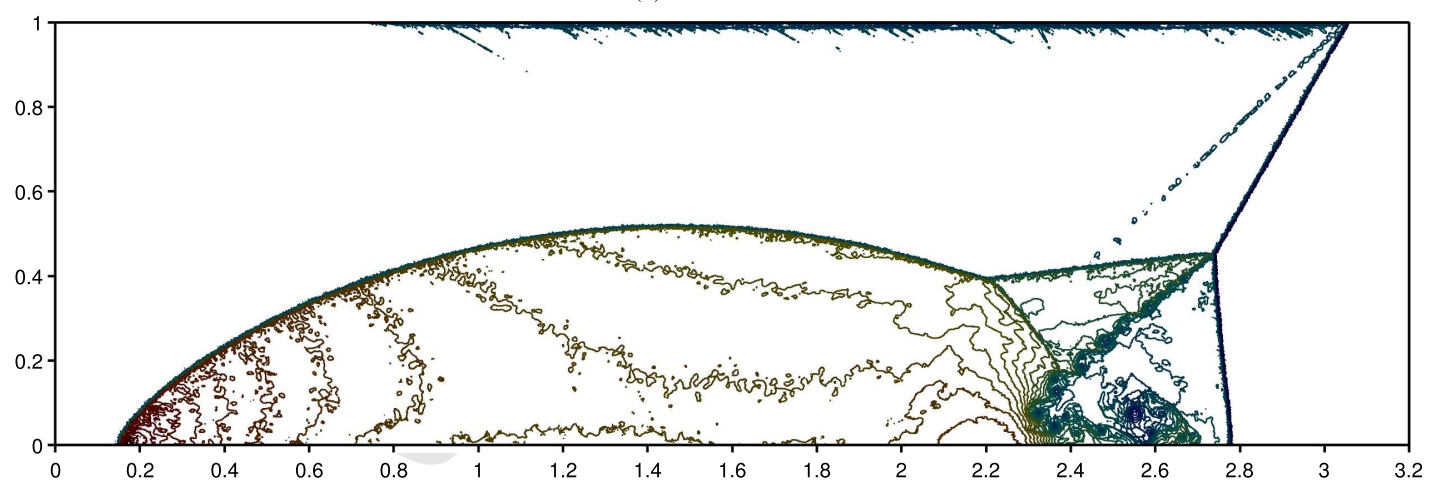

(d) fifth-order

Fig. 29. High-order DG with proposed WENO and positivity preserving with irregular triangular elements $(h=1 / 200)$ in $(x, y) \in[0,4] \times[0,1]$. Shown are 41 equidistance density contour lines at $t=0.2$ from 1.5 to 22.5 . 


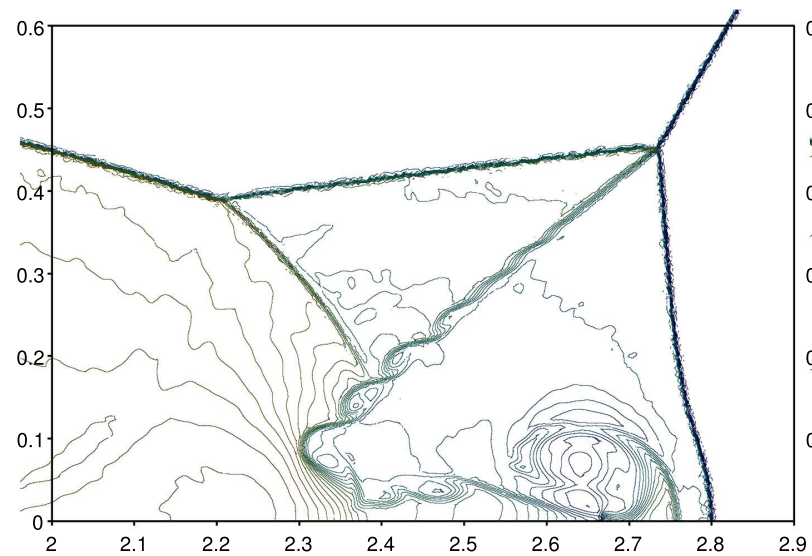

(a) second-order

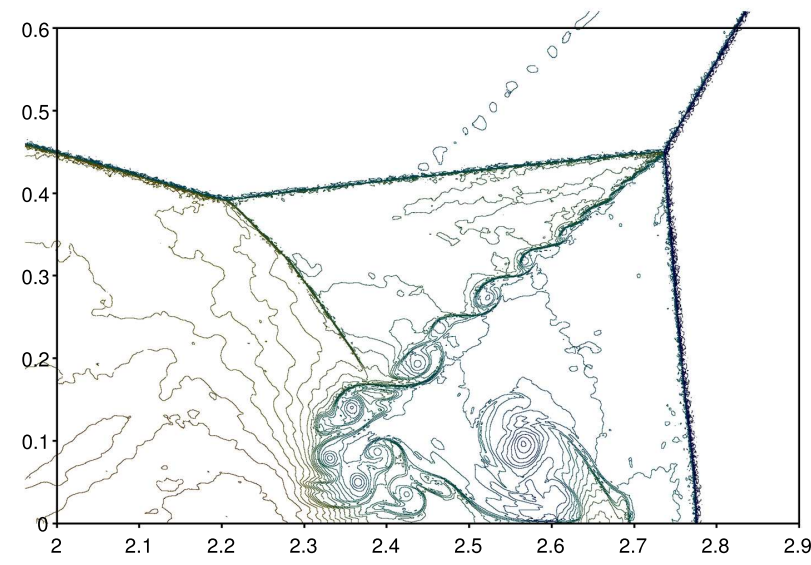

(c) fourth-order

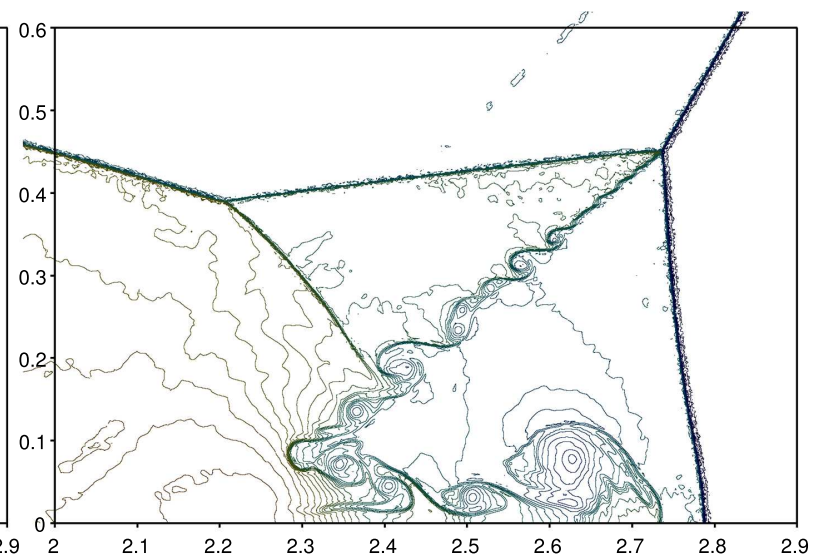

(b) third-order

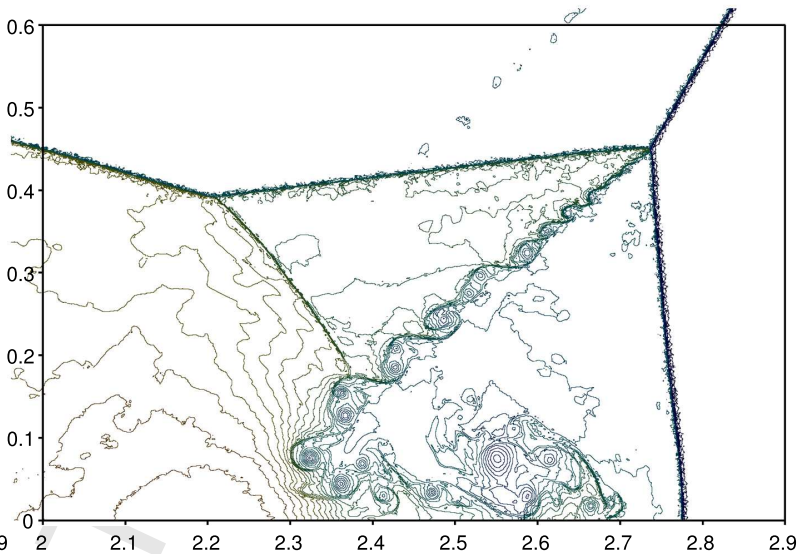

(d) fifth-order

Fig. 30. High-order DG with proposed WENO and positivity preserving with irregular triangular elements $(h=1 / 200)$ in $(x, y) \in[0,4] \times[0,1]$. Shown are zoom-in views around the Mach stems at $t=0.2$ with 41 equidistance density contour lines from 1.5 to 22.5 .

reducing the elemental residual to machine zero than the first proposed limiter. Both limiters however showed similar end results. The proposed limiter performed well in the Sod and the Lax problems. The limiter also performed remarkably well capturing the high-frequency oscillations generated by the shock-turbulence interactions in the Shu-Osher problem, as well as the complexity of the triple Mach point and the curled flow structures in the double Mach reflection problem. Practical problems such as the two-strut scramjet and blunt body problems were reasonably predicted by the proposed limiter. Blunt body surface quantiles showed nearly smooth solution predictions even in the presence of striation-like patterns in the post shock regions due to irregularities of the grid elements in the shock regions, and misalignment of the element faces with the bow shock. Further improvement to the WENO polynomial limiter, and extensions to three-dimensional tetrahedral elements will be reported in future studies.

\section{References}

[1] S. Gottlieb, C.-W. Shu, E. Tadmor, Strong stability-preserving high-order time discretization methods, SIAM Rev. 43 (2001) 89-112.

[2] S. Gottlieb, D.I. Ketcheson, C.-W. Shu, High order strong stability preserving time discretizations, J. Comput. Sci. 38 (2009) 251-289.

[3] B. Cockburn, C.-W. Shu, TVB Runge-Kutta local projection discontinuous Galerkin finite element method for conservation laws II. General framework, Math. Comput. 52 (1989) 411-435.

[4] B. Cockburn, S.-Y. Lin, C.-W. Shu, TVB Runge-Kutta local projection discontinuous Galerkin finite element method for conservation laws III. One dimensional systems, J. Comput. Phys. 84 (1989) 90-113.

[5] B. Cockburn, S. Hou, C.-W. Shu, The Runge-Kutta local projection discontinuous Galerkin finite element method for conservation laws IV: the multidimensional case, Math. Comput. 54 (1990) 545-581.

[6] B. Cockburn, C.-W. Shu, The Runge-Kutta discontinuous Galerkin method for conservation laws V, J. Comput. Phys. 141 (1998) $199-224$.

[7] A. Jameson, Numerical Solution of the Euler Equations by Finite Volume Methods Using Runge-Kutta Time-Stepping Schemes, AIAA Paper 1981-1259, 1981.

[8] R. Hartmann, Adaptive discontinuous Galerkin methods with shock-capturing for the compressible Navier-Stokes equations, Int. J. Numer. Methods Fluids 51 (2005) 1131-1156.

[9] P.-O. Persson, J. Peraire, Sub-cell shock capturing for discontinuous Galerkin methods, AIAA Paper 2006-112, 2006. 
[10] Y. Lv, Y.C. See, M. Ihme, An entropy-residual shock detector for solving conservation laws using high-order discontinuous Galerkin methods, J. Comput. Phys. 322 (2016) 448-472.

[11] H. Harten, High resolution schemes for hyperbolic conservation laws, J. Comput. Phys. 49 (1983) 357-393.

[12] S. Osher, C. Chakravarthy, High-resolution schemes and the entropy condition, SIAM J. Math. Anal. 21 (1984) 955-984.

[13] H.C. Yee, R.F. Warming, A. Harten, Implicit total variation diminishing (TVD) schemes for steady-state calculations, J. Comput. Phys. 57 (1985) $327-360$.

[14] J. Qiu, C.-W. Shu, Hermite WENO schemes and their application as limiters for Runger-Kutta discontinuous Galerkin method: one dimensional case, J. Comput. Phys. 193 (2003) 115-135.

[15] J. Qiu, C.-W. Shu, Runge-Kutta discontinuous Galerkin method using WENO limiters, SIAM J. Sci. Comput. 26 (2005) 907-927.

[16] J. Qiu, C.-W. Shu, Hermite WENO schemes and their application as limiters for Runge-Kutta discontinuous Galerkin method II: two dimensional case, Comput. Fluids 34 (2005) 642-663.

[17] X. Zhong, C.-W. Shu, A simple weighted essentially nonoscillatory limiter for Runge-Kutta discontinuous Galerkin method, J. Comput. Phys. 232 (2013) 397-415.

[18] J. Zhu, J. Qiu, Runge-Kutta discontinuous Galerkin method using WENO type limiters: Three dimensional unstructured meshes, Commun. Comput. Phys. 11 (2012) 985-1005.

[19] J. Zhu, X. Zhong, C.-W. Shu, J. Qiu, Runge-Kutta discontinuous Galerkin method using a new type of WENO limiters on unstructured meshes, J. Comput. Phys. 248 (2013) 200-220.

[20] G. Jiang, C.-W. Shu, Efficient implementation of weighted ENO schemes, J. Comput. Phys. 126 (1996) 202-228.

[21] C. Hu, C.-W. Shu, Weighted essentially non-oscillatory schemes on triangular meshes, J. Comput. Phys. 150 (1999) 97-127.

[22] J. Shi, C. Hu, C.-W. Shu, A technique of treating negative weights in WENO schemes, J. Comput. Phys. 175 (2002) 108-127.

[23] J. Zhu, J. Qui, C.-W. Shu, M. Dumbser, Runge-Kutta discontinuous Galerkin method using WENO limiters II: unstructured meshes, J. Comput. Phys. 227 (2008) 4330-4353.

[24] Y.-T. Zhang, C.-W. Shu, Third order WENO scheme on three dimensional tetra-hedral meshes, Commun. Comput. Phys. 5 (2009) $836-848$.

[25] J. Zhu, C.-W. Shu, On the convergence to steady state solution of a new class of high order WENO schemes, J. Comput. Phys. 349 (2017) 80-96.

[26] J. Zhu, C.-W. Shu, Numerical study on the convergence to steady state solutions of a new class of finite volume WENO schemes: triangular meshes, Shock Waves 29 (2019) 3-25.

[27] D. Levy, G. Puppo, G. Russo, Central WENO schemes for hyperbolic systems of conservation laws, M2AN. Math. Model. Numer. Anal. 33 (1999) 547-571.

[28] J. Zhu, J. Qiu, A new fifth order finite difference WENO scheme for solving hyperbolic conservation laws, J. Comput. Phys. 318 (2016) 110-121.

[29] M. Dumbser, R. Loubère, A simple robust and accurate a posteriori subcell finite volume limiter for the discontinuous Galerkin method on unstructured meshes, J. Comput. Phys. 319 (2016) 163-199.

[30] M. Dumbser, W. Boscheri, M. Semplice, G. Russo, Central WENO schemes for hyperbolic conservation laws on fixed and moving unstructured meshes, SIAM J. Sci. Comput. 39 (2017) A2564-A2591.

[31] J. Zhu, J. Qiu, A new third order finite volume weighted essentially non-oscillatory scheme on tetrahedral meshes, J. Comput. Phys. 349 (2017) $220-232$.

[32] C. Wang, X. Zhang, C.-W. Shu, J. Ning, Robust high order discontinuous Galerkin schemes for two-dimensional gaseous detonations, J. Comput. Phys. 231 (2012) 653-665.

[33] J. Zhu, J. Qiu, A new type of finite volume WENO schemes for hyperbolic conservation laws, J. Sci. Comput. 73 (2017) 1338-1359.

[34] J. Zhu, J. Qiu, New finite volume weighted essentially non-oscillatory schemes on triangular meshes, SIAM J. Sci. Comput. 40 (2018) A903-A928.

[35] R. Borges, M. Carmona, B. Costa, W.S. Don, An improved weighted essentially non-oscillatory scheme for hyperbolic conservation laws, J. Comput. Phys. 227 (2008) 3191-3211.

[36] M. Castro, B. Costa, W.S. Don, High order weighted essentially non-oscillatory WENO-Z schemes for hyperbolic conservation laws, J. Comput. Phys. 230 (2011) $1766-1792$

[37] W.S. Don, R. Borges, Accuracy of the weighted essentially non-oscillatory conservative finite difference schemes, J. Comput. Phys. 250 (2013) $347-372$.

[38] O. Kolb, On the full and global accuracy of a compact third order weno scheme, SIAM J. Numer. Anal. 52 (2014) 2335-2355.

[39] P. Woodward, P. Colella, The numerical simulation of two-dimensional fluid flow with strong shocks, J. Comput. Phys. 54 (1984) 115-173.

[40] B. Cockburn, C.-W. Shu, Runge-Kutta discontinuous Galerkin methods for convection-dominated problems, J. Comput. Phys. 16 (2001) $173-261$.

[41] C.-W. Shu, S. Osher, Efficient implementation of essentially non-oscillatory shock-capturing schemes II, J. Comput. Phys. 93 (1989) 32-78.

[42] Ajay Kumar, Numerical Analysis of Scramjet-Inlet Flow Field by Using Two-Dimensional Navier-Stokes Equations, Technical Report TP-1981-1940, NASA, December 1981.

[43] A. Eberle, M. Schmatz, N. Bissinger, Generalized fluxvectors for hypersonic shock capturing, in: 28th AIAA Aerospace Science Meeting, AIAA Paper 1990-0390, Reno, NV, 1990

[44] Frédéric Alauzet, Pascal Frey, Estimateur dérreur géométrique et métriques anisotropes pour l'adaptation de maillage: Partie II: exemples d'applications, Technical Report PR-4789, INRIA, 2003.

[45] F. Kemm, On the proper setup of the double Mach reflection as a test case for the resolution of gas dynamics codes, Comput. Fluids 132 (2016) $72-75$.

[46] J. Zhu, X. Zhong, C.-W. Shu, J. Qiu, Runge-Kutta discontinuous Galerkin method with a simple and compact Hermite WENO limiter, Commun. Comput. Phys. 21 (2017) 623-649. 


\section{Sponsor names}

Do not correct this page. Please mark corrections to sponsor names and grant numbers in the main text.

NASA, country=United States, grants $=\mathrm{CIF} / \mathrm{IRAD}$

NASA, country=United States, grants=STMD ESM/GCD

ARO, country=United States, grants=W911NF-15-1-0226

NASA, country $=$ United States, grants $=\mathrm{CIF} / \mathrm{IRAD}$

1 


\section{Highlights}

- Compact high-order WENO for DG methods on triangular elements.

- Bounded polynomial limiter with a positivity preserving.

- $(k+1)$ th order of accuracy on irregular triangular elements.

- Nonintrusive and simple implementation procedures. 